\title{
Diversidade da ictiofauna do Alto Rio Paraná: composição atual e perspectivas futuras
}

\section{Francisco Langeani ${ }^{1,6}$, Ricardo Macedo Corrêa e Castro ${ }^{2}$, Osvaldo Takeshi Oyakawa ${ }^{3}$,} Oscar Akio Shibatta 4 , Carla Simone Pavanelli ${ }^{5} \&$ Lilian Casatti $^{1}$

Biota Neotropica v7 (n3) - http://www.biotaneotropica.org.br/v7n3/pt/abstract?article + bn03407032007

\author{
Recebido em 21/12/06 \\ Versão reformulada recebida em 06/07/07 \\ Publicado em 19/10/07
}

\author{
${ }^{1}$ Laboratório de Ictiologia, Departamento de Zoologia e Botânica, \\ Universidade Estadual Paulista - UNESP, Rua Cristóvão Colombo, 2265, CEP 15054-000, \\ São José do Rio Preto, SP, Brasil, e-mail: lcasatti@ibilce.unesp.br \\ ${ }^{2}$ Laboratório de Ictiologia de Ribeirão Preto - LIRP, Departamento de Biologia, \\ Faculdade de Filosofia Ciencias e Letras de Ribeirao Preto - FFCLRP, \\ Universidade de São Paulo - USP,
}

Av. Bandeirantes, 3900, CEP 14040-901, Ribeirão Preto, SP, Brasil, e-mail: rmcastro@ffclrp.usp.br ${ }^{3}$ Museu de Zoologia, Universidade de São Paulo, Av. Nazaré, 481, CP 42494, CEP 04218-970, São Paulo, SP, Brasil, e-mail: oyakawa@usp.br ${ }^{4}$ Departamento de Biologia Animal e Vegetal, Centro de Ciências Biológicas, Universidade Estadual de Londrina - UEL, Rodovia Celso Garcia Cid - PR 445, Km 380, CEP86051-990, Londrina,PR, Brasil, e-mail: shibatta@uel.br

${ }^{5}$ Núcleo de Pesquisas em Limnologia Ictiologia e Aqüicultura - NUPELIA, Universidade Estadual de Maringá,

Av. Colombo, 5790, CEP 87020-900, Maringá,PR, Brasil.e-mail: carlasp@nupelia.uem.br ${ }^{6}$ Autor para correspondência: Francisco Langeani, e-mail: langeani@ibilce.unesp.br

\begin{abstract}
Langeani, F., Castro, R.M.C., Oyakawa, O.T., Shibatta, O.A., Pavanelli, C.S. \& Casatti, L. Ichthyofauna diversity of the upper rio Paraná: present composition and future perspectives. Biota Neotrop. Sep/Dez 2007 vol. 7, no. 3 http://www.biotaneotropica.org.br/v7n3/pt/abstract?article+bn03407032007. ISSN 1676-0603.

A synthesis concerning fishes from Upper Paraná River basin is presented, based on data from fish-collections, literature, and new field samples. Three hundred and ten species, pertaining to 11 orders and 38 families, are referred to the drainage, elevating anterior estimates. Concerning total species, 236 (76.1\%) are autochthonous, 67 (21.6\%) are allochthonous, and seven (2.3\%) are exotic. Principal causes of occurrence of non-native species are: a) dispersal from the Lower Paraná, after the construction of Itaipu dam, and b) escapes from aquaculture farms. Most species $(65 \%)$ are small-sized, having less than $21 \mathrm{~cm}$ of length, and the great majority occurs only in headwaters and small streams. One of the best known and most studied in Brazil, Upper Paraná ichthyofauna richness is far from reaching actual numbers, considering the exponential elevation of species presented herein. Indeed, in the last years various new species have been described and around 50 other species, already recognized as new, are now under description. The improvement on the knowledge about Upper Paraná ichthyofauna is proportional to the number of researchers involved with studies in the area and, unequivocally, reflects recent initiatives stimulating and incrementing taxonomic research, and also improving access to fish collections and to poor or never-sampled areas. However, if we are going to maintain the number of species descriptions per year of last decade, the 50 new species already recognized, will be described only in the next ten years, a period too long. In consequence it is very important that scientific community and grant agencies find and offer initiatives in order to elevate the number of new taxa descriptions per year.
\end{abstract}

Keywords: fishes, freshwater, southeasten Brazil, inventory, transposition.

\section{Resumo}

Langeani, F., Castro, R.M.C., Oyakawa, O.T., Shibatta, O.A., Pavanelli, C.S. \& Casatti, L. Diversidade da ictiofauna do Alto Rio Paraná: composição atual e perspectivas futuras. Biota Neotrop. Sep/Dez 2007 vol. 7 , no. 3 http://www.biotaneotropica.org.br/v7n3/pt/abstract?article+bn03407032007. ISSN 1676-0603.

É apresentada uma síntese sobre os peixes do Alto Paraná, com base em dados de coleções, dados de literatura e novas coletas. Trezentas e dez espécies, de 11 ordens e 38 famílias, são referidas para a drenagem, aumentando significativamente números anteriores. Dentre as espécies da área, 236 (76,1\%) são autóctones, 67 (21,6\%) alóctones e sete $(2,3 \%)$ exóticas. As principais causas de ocorrência de espécies não nativas (alóctones e exóticas) foram a dispersão a partir do baixo Paraná, após a construção do Reservatório de Itaipu e o escape de pisciculturas. A maior parte das espécies referidas (65\%) tem porte pequeno, sendo menor que $21 \mathrm{~cm}$ de comprimento; dentre essas, a maioria 
ocorre apenas em riachos e cabeceiras. Apesar da ictiofauna do Alto Paraná ser uma das melhor conhecidas e mais estudadas, o número de espécies descritas ou referidas para a área tem crescido exponencialmente, o que indica que a riqueza apresentada está longe de representar a realidade. De fato, várias novas espécies têm sido descritas nos últimos anos e cerca de 50 novas espécies, já reconhecidas, estão em fase de descrição. A melhoria no conhecimento sobre a ictiofauna do Alto Paraná é proporcional ao número de pesquisadores envolvidos em estudos na bacia e reflete, de modo inequívoco, iniciativas recentes que têm estimulado e incrementado pesquisas taxonômicas, facilitado o acesso ao material depositado em coleções científicas e aumentado as coletas em áreas e ambientes pouco amostrados. Entretanto, mantido o ritmo de descrições de novas espécies ocorrido até agora nessa última década, as 50 novas espécies já reconhecidas estariam descritas apenas dentro de dez anos, um tempo demasiadamente longo. Por essa razão é muito importante que a comunidade científica e os órgãos de fomento encontrem e viabilizem iniciativas de modo a aumentar esse ritmo de descrições de novos táxons e disponibilizar esses novos nomes mais rapidamente.

Palavras-chave: peixes, água doce, sudeste brasileiro, inventário, transposição.

\section{Introdução}

Hoje são conhecidas aproximadamente 1,8 milhão de espécies de organismos vivos (Cox \& Moore 2000), dos quais aproximadamente 55.000 são vertebrados e, dentre esses, aproximadamente 28.000 são peixes (Nelson 2006). A grande riqueza de espécies de peixes reflete-se também na sua diversidade morfológica e ecológica. A maior parte dessa riqueza e diversidade encontra-se em águas tropicais (Lowe-McConnell 1999), particularmente nas águas doces neotropicais, habitadas por 4.475 espécies válidas de peixes, podendo chegar a mais de 6.000 (dentre as 13.000 mundiais) se incluídas as novas espécies já reconhecidas por especialistas, porém ainda não descritas (Reis et al. 2003).

Na Região Neotropical, a América do Sul abriga a maior parte dessa diversidade nas bacias Amazônica e do Paraná; a primeira com uma área de cerca de $7.000 .000 \mathrm{~km}^{2}$ e entre 1.500 e 5.000 espécies de peixes (Santos \& Ferreira 1999); a segunda, com cerca de $2.600 .00 \mathrm{~km}^{2}$ (ou 2.985.000 se incluirmos o rio Uruguai) (Latrubesse et al. 2005) e aproximadamente 600 espécies (Bonetto 1986). Para a porção do Alto Paraná com 900.000 km² (Figura 1), há estimativas variando de 130 espécies (Bonetto 1986) a mais de 250 apenas no trecho brasileiro da bacia (Agostinho \& Júlio-Jr 1999). Para o Estado de São Paulo são referidas 166 espécies (Castro \& Menezes 1998).

Inventários recentes em ambientes de riachos e de cabeceiras no Alto Paraná, principalmente do Estado de São Paulo (e.g., Casatti et al. 2001, Castro \& Casatti 1997, Castro et al. 2003, 2004, 2005, Langeani et al. 2005a,b), comprovam a ocorrência de uma fauna bastante diver-

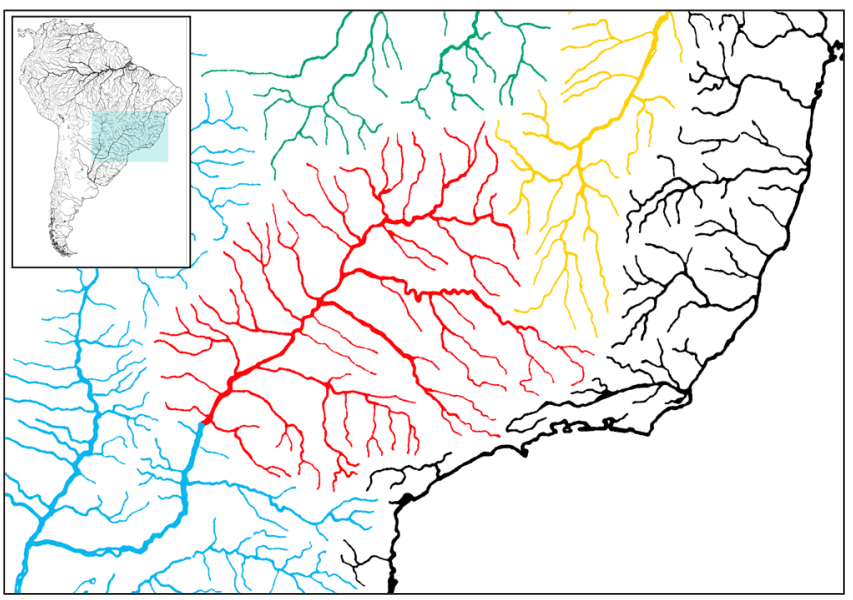

Figura 1. Sistema do Alto rio Paraná (vermelho) e bacias vizinhas do Paraguai e Baixo Paraná (azul), Araguaia/Tocantins (verde), São Francisco (amarelo) e rios costeiros (preto).

Figure 1. Upper Rio Paraná system (red) and neighbor basins of Paraguay and Low Paraná (blue), Araguaia/Tocantins (green), São Francisco (yellow) and coastal rivers (black). sificada, além de registrar a ocorrência de várias espécies alóctones e exóticas e mostrar que cerca de 6 a $15 \%$ das espécies referidas são novas (Castro et al. 2003, 2004, 2005). Resultados semelhantes têm sido obtidos também por meio da revisão de coleções ictiológicas e de outros estudos independentes, reforçando o fato de que os levantamentos realizados no Alto Paraná são incompletos (Agostinho \& Gomes 2005) e mostrando a importância de se incrementar esforços de coleta na área e de se revisar o material depositado em coleções.

É apresentada aqui uma síntese sobre a diversidade da ictiofauna do Alto Paraná, com base em dados das coleções ictiológicas da Universidade Estadual de Londrina (MZUEL); Universidade Estadual de Maringá (NUP); Universidade Estadual Paulista, UNESP, campus de São José do Rio Preto (DZSJRP); Universidade de São Paulo, campus de Ribeirão Preto (LIRP) e Museu de Zoologia da Universidade de São Paulo (MZUSP), além de informações mais recentes provenientes de literatura especializada.

\section{Caracterização da Área}

O Sistema do Alto Rio Paraná (doravante Alto Paraná) (Figura 1) inclui toda a drenagem do Rio Paraná à montante do antigo Salto de Sete Quedas (agora inundado pelo Reservatório de Itaipu) (Bonetto 1986, Britski \& Langeani 1988). Com aproximadamente 900 mil km², essa porção da bacia faz parte da face sul do Escudo Brasileiro e é representada por domínios morfoclimáticos que incluem Florestas Estacionais Semideciduais, Cerrados, Florestas Ombrófilas Mistas, Campos Rupestres e Matas de Galeria (Hueck \& Seibert, 1981).

Os principais rios da margem esquerda do rio Paraná nascem em rochas cristalinas da Serra do Mar enquanto que aqueles da margem direita nascem nas Serras de Maracaju e do Carapó (Souza Filho \& Stevaux 1997). A porção sudeste do Escudo Cristalino Brasileiro abriga as cabeceiras de seus formadores e afluentes, os rios Grande, Paranaíba, Paranapanema e Tietê, bem como as cabeceiras de bacias adjacentes, tais como dos rios Tocantins-Araguaia, Doce, Paraíba do Sul, Ribeira de Iguape, São Francisco e diversas drenagens litorâneas menores.

Em razão da ocorrência de rochas cristalinas o relevo é acidentado na porção leste e sudeste da bacia do Alto Paraná. Nas demais áreas predominam formas tabulares onduladas, com suave inclinação em direção ao rio Paraná, interrompidas pelas escarpas da Serra Geral, formando o que é atualmente chamado de planalto central da bacia do Paraná, com altitudes que variam de 500 a 1.000 metros (Souza Filho \& Stevaux 1997).

\section{Biogeografia}

O Alto Paraná é área complexa devido às atividades tectônicas pelas quais tem passado desde o início do Terciário (Ab'Saber 1998). Essas atividades, associadas ao complexo sistema de falhas 
existentes na área, são a principal causa de diversos eventos de captura de cabeceiras, como ocorrido entre os rios Tietê e Paraíba do Sul (Castro et al. 2003), e que foram responsáveis pela distribuição de algumas de suas espécies também em drenagens vizinhas, tais como: rios Paraíba do Sul, Ribeira de Iguape e algumas drenagens litorâneas menores (Langeani 1989, Weitzman \& Malabarba 1999, Ribeiro 2006, Ribeiro et al. 2006, Serra et al. 2007), ou ainda no Rio São Francisco (Britski et al. 1988, Britto \& Castro 2002). Malabarba (1998) sugere a existência de conexões pretéritas entre o rio Tietê e drenagens costeiras através de uma conformação antiga do vale do rio Paraíba; essas conexões podem também ter ocorrido em outras porções do Alto Paraná, o que precisaria ser melhor investigado através de estudos filogenéticos e biogeográficos dos grupos de peixes que ocorrem nessas bacias e também por meio de evidências geológicas desses eventos de captura de drenagens.

Sob o ponto de vista ictiofaunístico, portanto, o Alto Paraná compreende uma área com história própria complexa e também, em parte, compartilhada com drenagens vizinhas. Além disso, apresenta um inequívoco endemismo (e.g., Britski \& Langeani 1988, Castro et al. 2003, Langeani 1989, Vari 1988, Menezes, Weitzman \& Burns 2003), sendo caracterizado como uma província ictiofaunística natural (Géry 1969).

\section{Material e Métodos}

A lista de espécies foi elaborada a partir do exame das coleções de peixes da UNESP de São José do Rio Preto (DZSJRP), Museu de Zoologia da USP (MZUSP), Laboratório de Ictiologia de Ribeirão Preto, USP (LIRP), coleções cujos dados estão parcial ou totalmente disponíveis em http://splink.cria.org.br/, Universidade Estadual de Londrina (MZUEL) e Universidade Estadual de Maringá (NUP). A lista de espécies foi confrontada com os dados de "Check list of the freshwater fishes of South and Central America" (Reis et al., 2003), Fishbase (http://filaman.ifm-geomar.de/) ou "Catalog of Fishes" (Eschmeyer, 1998; http://www.calacademy.org/research/ichthyology/ catalog/fishcatsearch.html). Também, partes da lista foram enviadas a especialistas em cada um dos grupos taxonômicos para confirmação das espécies referidas, adição de espécies, informações sobre a distribuição das espécies no Alto Paraná e sobre prováveis novas espécies, ainda não descritas. Foram incluídas todas as espécies descritas ou referidas para o Alto Paraná em literatura, até dezembro de 2006.

Material testemunho listado baseia-se em exemplares examinados pelos autores ou por especialistas consultados (ver Agradecimentos). Exceções incluem alguns tipos primários de espécies não representadas nas coleções examinadas.

As comparações e análises realizadas consideraram autóctones (nativas) as espécies que ocorrem naturalmente no Alto Paraná, incluindo em alguns casos espécies descritas com base em material tipo proveniente de outras bacias. As espécies alóctones são aquelas descritas de outras bacias da Região Neotropical e introduzidas no Alto Paraná, sem quaisquer evidências que possam indicar sua ocorrência natural no Alto Paraná. Finalmente, espécies exóticas são aquelas provenientes de outros continentes. As espécies alóctones e exóticas foram classificadas, também segundo a possível causa de sua ocorrência no Alto Paraná, a saber: itaipu, espécies cujos primeiros registros na área são posteriores à construção do Reservatório de Itaipu, o que possibilitou sua dispersão pelo Alto Paraná; piscicultura, espécies amplamente utilizadas em pisciculturas da região, introduzidas intencional ou acidentalmente; pesca, espécies introduzidas para a pesca esportiva ou para uso como iscas para a pesca esportiva; aquarismo, espécies de aquário introduzidas intencional ou acidentalmente; controle de mosquitos, espécies introduzidas para o controle de mosquitos; desconhecida, espécies cuja causa de ocorrência é desconhecida. Uma revisão recente sobre introdução de espécies em águas brasileiras pode ser consultada em Agostinho et al. (2007, cap. 6), onde os autores comentam sobre as espécies mais freqüentes e razões e finalidades das introduções.

De modo a avaliar a composição em tamanho da ictiofauna do Alto Paraná, o tamanho máximo de cada espécie (comprimento padrão, comprimento total ou comprimento do disco) foi compilado de Reis et al. (2003), de Fishbase (http://www.fishbase.org ) ou de descrições originais. Os valores encontrados $(n=307)$ foram divididos em classes de tamanho, obtidas através da fórmula de Sturges $(\mathbf{K}=\mathbf{1}+\mathbf{3 , 3 2} \log \mathbf{n}$, sendo $\mathbf{K}$ o número de classes e $\mathbf{n}$ o tamanho da amostra) que dá o número de classes para a amostra analisada, sendo que o tamanho do intervalo das classes é calculado pela amplitude de tamanho dividida pelo número de classes.

\section{Resultados e Discussão}

\section{Espécies}

O Alto Paraná abriga 310 espécies de peixes (Tabela 1), distribuídas em 11 ordens (Figura 2) e 38 famílias (Figura 3). Esse número de espécies é significativamente maior que referências anteriores que apontavam desde 130 espécies (Bonetto 1986), passando por 166 espécies (Castro \& Menezes 1998), até mais de 250 espécies (Agostinho \& Júlio-Jr. 1999). É importante ressaltar, entretanto, que Castro \& Menezes (1998) referiram-se apenas às espécies do Alto Paraná no Estado de São Paulo, enquanto a lista de Agostinho \& Júlio-Jr. (1999) com 231 espécies (apesar de fazerem referência a mais de 250 espécies no texto), inclui espécies não identificadas e outras provenientes de trecho à jusante do Reservatório de Itaipu e também do rio Iguaçu à montante das cataratas, porções não pertencentes ao Alto Paraná como considerado aqui. Importa notar ainda que a barragem de Itaipu, barreira para o trecho que hoje corresponde ao Alto Paraná, está localizada 150 km à jusante do Salto de Sete Quedas, a antiga barreira natural entre as porções do alto e médio/baixo rio Paraná. O efeito eclusa desta nova barreira, que reuniu parte do trecho do Baixo Paraná com o Alto Paraná, contribuiu significativamente para o aumento do número de espécies do Alto Paraná, conforme já relatado por Agostinho \& Júlio-Jr. (1999).

A maior riqueza é registrada em Siluriformes e Characiformes, que respondem por cerca de $80 \%$ das espécies e compõem os grupos dominantes na maior parte dos ambientes lóticos do Alto Paraná. Britski (1992) afirma que os Otophysi (Characiformes, Gymnotiformes e Siluriformes) são os responsáveis por mais de $90 \%$ das espécies, o que não corresponde aos valores encontrados aqui (aproximadamente $85 \%$ ), conseqüência de um grande número de Cyprinodontiformes descritos recentemente, bem como de ciclídeos (Cichlidae, Perciformes), mais abundantes em ambientes de águas mais calmas. Nesses ambientes lênticos, representados no Alto Paraná principalmente pelos reservatórios artificiais, tem havido um aumento considerável de espécies alóctones ou exóticas de Perciformes (Lowe-McConnell 1999), principalmente Cichlidae, que eventualmente podem mostrar dominância, e Cyprinodontiformes, aliado a um decréscimo de espécies autóctones, principalmente de Siluriformes.

Quanto à origem da fauna (Figura 4), 236 espécies $(76,1 \%)$ são autóctones, $67(21,6 \%)$ são alóctones e sete $(2,3 \%)$ são exóticas. Dentre as alóctones e exóticas, 37 passaram a ocorrer no Alto Paraná depois da construção da barragem de Itaipu, 13 possuem causa de ocorrência desconhecida, dez são espécies utilizadas em piscicultura, cinco são espécies utilizadas para a pesca esportiva ou como isca nesse mesmo tipo de pesca, quatro são espécies de aquário, duas são espécies utilizadas para controle de mosquitos, uma de aquário/pisci- 
Tabela 1. Ictiofauna do Alto rio Paraná: espécies, voucher (número de registro do lote testemunho), tam. (comprimento padrão, comprimento total * ou largura do disco**) em centímetros, origem da espécie e causa de sua ocorrência no Alto Paraná.

Table 1. Upper rio Paraná fish species: voucher (number of testimony lot), tam. - size (standard length, total length* or disc width**) in centimeters, origem - species origin, and cause of occurrence in the Upper Paraná.

\begin{tabular}{|c|c|c|c|c|c|}
\hline & Espécie & Voucher $^{1}$ & Tam. (cm) & Origem $^{2}$ & Ocorrência $^{3}$ \\
\hline & MYLIOBATIFORMES & & & & \\
\hline & Potamotrygonidae & & & & \\
\hline 1 & Potamotrygon falkneri Castex \& Maciel, 1963 & 4802 & $47 * *$ & alóctone & Itaipu \\
\hline \multirow[t]{3}{*}{2} & Potamotrygon motoro (Müller \& Henle, 1841) & 4530 & $50 * *$ & alóctone & Itaipu \\
\hline & CYPRINIFORMES & & & & \\
\hline & Cyprinidae & & & & \\
\hline 3 & Platanichthys platana (Regan, 1917) & MZUSP92429 & 6,7 & alóctone & desconhecida \\
\hline 4 & Aristichthys nobilis (Richardson, 1845) & \# & 112 & exótica & piscicultura \\
\hline 5 & Ctenopharyngodon idella (Valenciennes, 1844) & NUP3633 & 15 & exótica & piscicultura \\
\hline \multirow[t]{3}{*}{6} & Cyprinus carpio Linnaeus, 1758 & NUP854 & 120 & exótica & piscicultura \\
\hline & Characiformes & & & & \\
\hline & Acestrorhynchidae & & & & \\
\hline \multirow[t]{2}{*}{7} & Acestrorhynchus lacustris (Lütken, 1875) & 5963 & 27 & autóctone* & nativa \\
\hline & Anostomidae & & & & \\
\hline 8 & Leporellus vittatus (Valenciennes, 1850) & NUP4211 & 24,5 & autóctone & nativa \\
\hline 9 & Leporinus aguapeiensis Campos, 1945 & MZUSP3040 & 18,8 & autóctone & nativa \\
\hline 10 & Leporinus amblyrhynchus Garavello \& Britski, 1987 & 4525 & 19,6 & autóctone & nativa \\
\hline 11 & Leporinus elongatus Valenciennes, 1850 & 750 & 50 & autóctone* & nativa \\
\hline 12 & Leporinus friderici (Bloch, 1794) & 3569 & 40 & autóctone* & nativa \\
\hline 13 & Leporinus lacustris Campos, 1945 & 3697 & 11,1 & autóctone & nativa \\
\hline 14 & Leporinus macrocephalus Garavello \& Britski, 1988 & 5145 & 40 & alóctone & piscicultura \\
\hline 15 & Leporinus microphthalmus Garavello, 1989 & 5397 & 11,8 & autóctone & nativa \\
\hline 16 & Leporinus obtusidens (Valenciennes, 1836) & 5466 & 40 & autóctone* & nativa \\
\hline 17 & Leporinus octofasciatus Steindachner, 1915 & 61 & 23,5 & autóctone* & nativa \\
\hline 18 & Leporinus paranensis Garavello \& Britski, 1987 & 22 & 16 & autóctone & nativa \\
\hline 19 & Leporinus striatus Kner, 1859 & 3415 & 25 & autóctone & nativa \\
\hline 20 & Leporinus tigrinus Borodin, 1929 & NUP1093 & - & autóctone* & nativa \\
\hline 21 & Schizodon altoparanae Garavello \& Britski, 1990 & 5258 & 23 & autóctone & nativa \\
\hline 22 & Schizodon borellii (Boulenger, 1900) & NUP1470 & 30 & autóctone* & nativa \\
\hline 23 & Schizodon intermedius Garavello \& Britski, 1990 & 4504 & 28,7 & autóctone & nativa \\
\hline \multirow[t]{3}{*}{24} & Schizodon nasutus Kner, 1858 & 6243 & 30 & autóctone & nativa \\
\hline & CHARACIDAE & & & & \\
\hline & Aphyocharacinae & & & & \\
\hline 25 & Aphyocharax anisitsi Eigenmann \& Kennedy, 1903 & 7531 & 5,5 & alóctone & Itaipu \\
\hline \multirow[t]{2}{*}{26} & Aphyocharax dentatus Eigenmann \& Kennedy, 1903 & 5324 & 6,9 & autóctone* & nativa \\
\hline & Bryconinae & & & & \\
\hline 27 & Brycon hilarii (Valenciennes, 1903) & NUP1748 & 41,5 & alóctone & desconhecida \\
\hline 28 & Brycon nattereri Günther, 1864 & 4408 & 29 & autóctone & nativa \\
\hline \multirow[t]{2}{*}{29} & $\begin{array}{l}\text { Brycon orbignyanus (Valenciennes in Cuvier \& Valenciennes, } \\
1850 \text { ) }\end{array}$ & 2302 & 79,5 & autóctone* & nativa \\
\hline & Characinae & & & & \\
\hline 30 & Cynopotamus kincaidi (Schultz, 1950) & NUP2025 & 17,4 & alóctone & Itaipu \\
\hline 31 & Galeocharax knerii (Steindachner, 1879) & 4474 & 22 & autóctone & nativa \\
\hline \multirow[t]{2}{*}{32} & Roeboides descalvadensis Fowler, 1932 & 4761 & 8,9 & alóctone & Itaipu \\
\hline & Cheirodontinae & & & & \\
\hline 33 & Aphyocheirodon hemigrammus Eigenmann, 1915 & 9330 & $4,8 *$ & autóctone & nativa \\
\hline 34 & Kolpotocheirodon theloura Malabarba \& Weitzman, 2000 & 10831 & 3 & autóctone & nativa \\
\hline 35 & Odontostilbe microcephala Eigenmann, 1907 & 3055 & 4,6 & autóctone* & nativa \\
\hline 36 & Serrapinnus heterodon (Eigenmann, 1915) & 2012 & 4,1 & autóctone & nativa \\
\hline 37 & Serrapinnus notomelas (Eigenmann, 1915) & 4883 & 3,6 & autóctone & nativa \\
\hline \multirow[t]{2}{*}{38} & Spintherobolus papilliferus Eigenmann, 1911 & 2244 & 6,1 & autóctone & nativa \\
\hline & Glandulocaudinae & & & & \\
\hline 39 & Glandulocauda melanogenys Eigenmann, 1911 & MZUSP28849 & 4,3 & autóctone & nativa \\
\hline 40 & Lophiobrycon weitzmani Castro; Ribeiro; Benine \& Melo, 2003 & LIRP4366Hol & 2,9 & autóctone & nativa \\
\hline 41 & Mimagoniates microlepis (Steindachner, 1876) & MZUEL4525 & 6,1 & autóctone* & nativa \\
\hline
\end{tabular}


Tabela 1. Continuação...

\begin{tabular}{|c|c|c|c|c|c|}
\hline & Espécie & Voucher $^{1}$ & Tam. $(\mathbf{c m})$ & Origem $^{2}$ & Ocorrência $^{3}$ \\
\hline & \multicolumn{5}{|l|}{ Stervardiinae } \\
\hline 42 & Planaltina britskii Menezes; Weitzman \& Burns, 2003 & 5439 & 3,6 & autóctone & nativa \\
\hline 43 & Planaltina glandipedis Menezes; Weitzman \& Burns, 2003 & 659 & 2,9 & autóctone & nativa \\
\hline 44 & Planaltina myersi Böhlke, 1954 & 10802 & 4,6 & autóctone & nativa \\
\hline 45 & Pseudocorynopoma heterandria Eigenmann, 1914 & MZUSP28756 & 5,6 & autóctone* & nativa \\
\hline \multicolumn{6}{|c|}{ Serrasalminae } \\
\hline 46 & Colossoma macropomum (Cuvier, 1818) & NUP1228 & $99,5^{*}$ & alóctone & piscicultura \\
\hline 47 & Metynnis maculatus (Kner, 1858) & 3725 & 18 & alóctone & Itaipu \\
\hline 48 & Metynnis mola Eigenmann \& Kennedy, 1903 & 4369 & 15 & alóctone & Itaipu \\
\hline 49 & Myleus tiete (Eigenmann \& Norris, 1900) & 654 & 15,2 & autóctone & nativa \\
\hline 50 & Mylossoma duriventre (Cuvier, 1818) & NUP2158 & 25 & autóctone* & nativa \\
\hline 51 & Piaractus mesopotamicus (Holmberg, 1887) & 6227 & 40,5 & autóctone* & nativa \\
\hline 52 & Serrasalmus maculatus Kner, 1858 & 629 & 20,2 & autóctone* & nativa \\
\hline 53 & Serrasalmus marginatus Valenciennes, 1837 & 6258 & 22,1 & autóctone & nativa \\
\hline \multicolumn{6}{|c|}{ Characidae Incertae Sedis } \\
\hline 54 & Astyanax altiparanae Garutti \& Britski, 2000 & 3712 & 5,7 & autóctone & nativa \\
\hline 55 & Astyanax biotae Castro \& Vari, 2004 & 7510 & 5,2 & autóctone & nativa \\
\hline 56 & Astyanax eigenmanniorum (Cope, 1894) & 6506 & 7,1 & autóctone* & nativa \\
\hline 57 & Astyanax fasciatus (Cuvier, 1819) & 3100 & $10^{*}$ & autóctone* & nativa \\
\hline 58 & Astyanax goyacensis Eigenmann, 1908 & MCZ20939Hol & 7,6 & autóctone & nativa \\
\hline 59 & Astyanax paranae Eigenmann, 1914 & 6326 & 13,9 & autóctone & nativa \\
\hline 60 & Astyanax paranahybae Eigenmann, 1911 & FMNH54714Hol & 5,4 & autóctone & nativa \\
\hline 61 & Astyanax schubarti Britski, 1964 & 2162 & 9 & autóctone & nativa \\
\hline 62 & Astyanax trierythropterus Godoy, 1970 & MZUSP16502 & 3,6 & autóctone & nativa \\
\hline 63 & Bryconamericus exodon Eigenmann, 1907 & NUP3673 & $5,7 *$ & alóctone & Itaipu \\
\hline 64 & Bryconamericus iheringii (Boulenger, 1887) & 8400 & 7,3 & autóctone* & nativa \\
\hline 65 & Bryconamericus stramineus Eigenmann, 1908 & 3574 & 5,6 & autóctone & nativa \\
\hline 66 & $\begin{array}{l}\text { Bryconamericus turiuba Langeani; Lucena; Pedrini \& Tarelho- } \\
\text { Pereira, } 2005\end{array}$ & 3425 & 6,1 & autóctone & nativa \\
\hline 67 & “Cheirodon" stenodon Eigenmann, 1915 & 3743 & 3,3 & autóctone & nativa \\
\hline 68 & Coptobrycon bilineatus (Ellis, 1911) & MZUSP4516 & 4,1 & autóctone & nativa \\
\hline 69 & Creagrutus varii Ribeiro; Benine \& Figueiredo, 2004 & 5424 & 4,2 & autóctone & nativa \\
\hline 70 & Gymnocorymbus ternetzi (Boulenger, 1895) & MZUSP49501 & 6 & alóctone & aquarismo \\
\hline 71 & Hasemania crenuchoides Zarske \& Géry, 1999 & MZUSP52732Hol & 6,7 & autóctone & nativa \\
\hline 72 & Hasemania hanseni (Fowler, 1949) & NUP1120 & 3,1 & autóctone & nativa \\
\hline 73 & Hemigrammus marginatus Ellis, 1911 & 4762 & 4,5 & autóctone* & nativa \\
\hline 74 & Hollandichthys multifasciatus (Eigenmann \& Norris, 1900) & 5724 & 9,6 & autóctone* & nativa \\
\hline 75 & Hyphessobrycon anisitsi (Eigenmann, 1907) & 330 & $5,7 *$ & autóctone* & nativa \\
\hline 76 & Hyphessobrycon balbus Myers, 1927 & 10820 & $6^{*}$ & autóctone & nativa \\
\hline 77 & Hyphessobrycon bifasciatus Ellis, 1911 & 6203 & $4,7 *$ & autóctone* & nativa \\
\hline 78 & Hyphessobrycon coelestinus Myers, 1929 & 7905 & $2,9 *$ & autóctone & nativa \\
\hline 79 & Hyphessobrycon duragenys Ellis, 1911 & MZUSP35239 & $6,8^{*}$ & autóctone & nativa \\
\hline 80 & Hyphessobrycon eques (Steindachner, 1882) & 5072 & 3,1 & autóctone* & nativa \\
\hline 81 & Hyphessobrycon flammeus Myers, 1924 & MZUSP86925 & 2,5 & alóctone & aquarismo \\
\hline 82 & Hyphessobrycon melanopleurus Ellis, 1911 & FMNH54413Hol & 3,5 & autóctone & nativa \\
\hline 83 & Hyphessobrycon reticulatus Ellis, 1911 & 5719 & 4,9 & autóctone* & nativa \\
\hline 84 & Knodus moenkhausii (Eigenmann \& Kennedy, 1903) & 4766 & $4,5^{*}$ & alóctone & Itaipu \\
\hline 85 & Moenkhausia intermedia Eigenmann, 1908 & 1923 & 8 & autóctone* & nativa \\
\hline 86 & Moenkhausia sanctaefilomenae (Steindachner, 1907) & 433 & 7 & autóctone* & nativa \\
\hline 87 & Oligosarcus paranensis Menezes \& Géry, 1983 & 2253 & 20,5 & autóctone & nativa \\
\hline 88 & Oligosarcus pintoi Campos, 1945 & 4746 & 8,4 & autóctone & nativa \\
\hline 89 & Oligosarcus planaltinae Menezes \& Géry, 1983 & 10806 & 9,9 & autóctone & nativa \\
\hline 90 & Piabina anhembi Silva \& Kaefer, 2003 & MZUSP59144Par & 7,9 & autóctone & nativa \\
\hline 91 & Piabina argentea Reinhardt, 1867 & 456 & 6,8 & autóctone* & nativa \\
\hline \multicolumn{6}{|c|}{ Salmininae } \\
\hline 92 & Salminus brasiliensis (Cuvier, 1816) & 7672 & 100 & autóctone* & nativa \\
\hline 93 & Salminus hilarii Valenciennes, 1850 & 5432 & 50 & autóctone* & nativa \\
\hline \multicolumn{6}{|c|}{ Triportheinae } \\
\hline 94 & Triportheus nematurus (Kner, 1858) & 624 & 15,9 & alóctone & Itaipu \\
\hline
\end{tabular}


Tabela 1. Continuação...

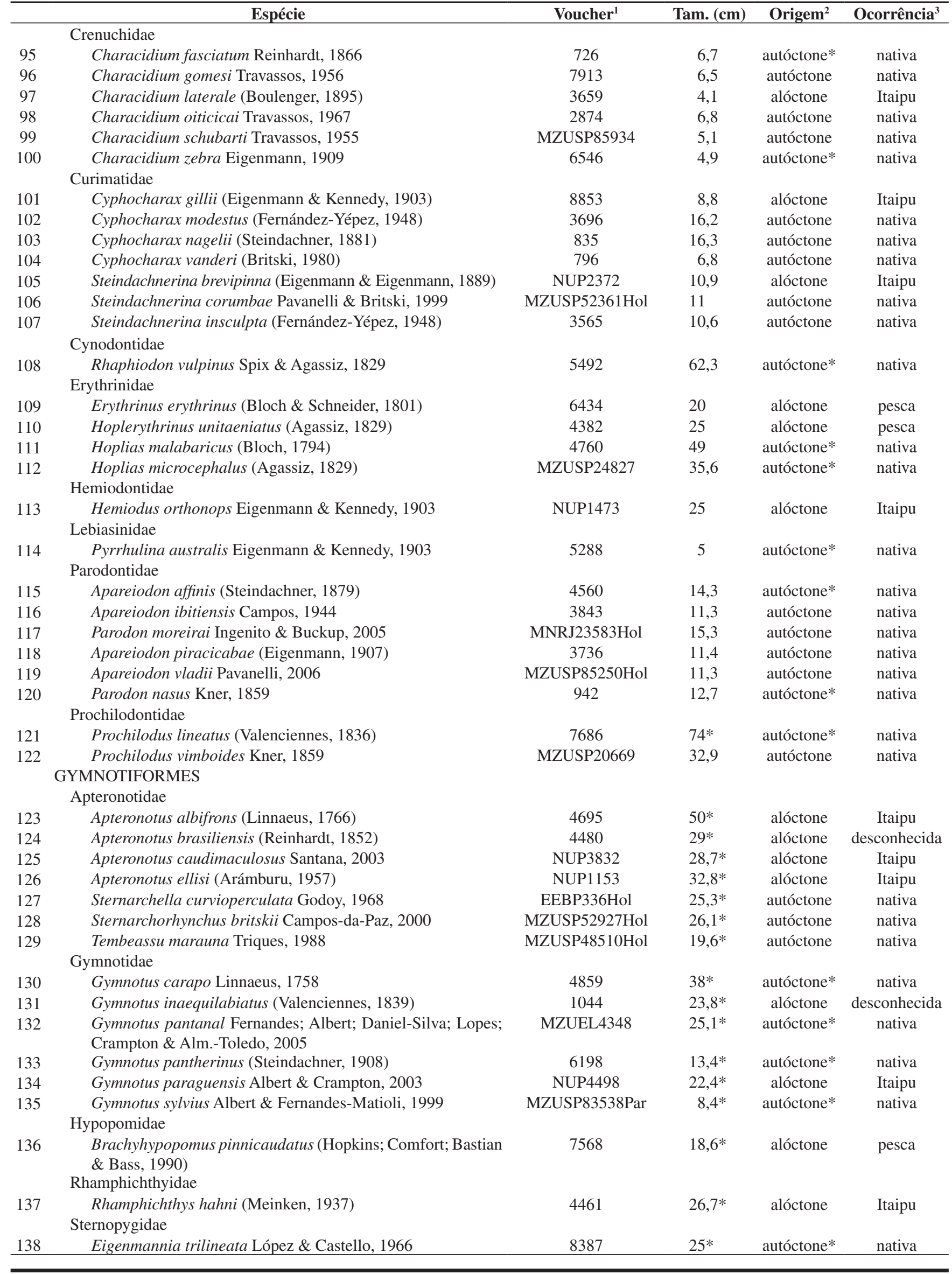


Tabela 1. Continuação...

\begin{tabular}{|c|c|c|c|c|c|}
\hline & Espécie & Voucher $^{1}$ & Tam. (cm) & Origem $^{2}$ & Ocorrência $^{3}$ \\
\hline 139 & Eigenmannia virescens (Valenciennes, 1847) & 5505 & $35 *$ & autóctone* & nativa \\
\hline \multirow[t]{2}{*}{140} & Sternopygus macrurus (Bloch \& Schneider, 1801) & 7477 & $140,5^{*}$ & autóctone* & nativa \\
\hline & $\begin{array}{l}\text { SILURIFORMES } \\
\text { Aspredinidae }\end{array}$ & & & & \\
\hline 141 & $\begin{array}{l}\text { Bunocephalus larai Ihering, } 1930 \\
\text { Auchenipteridae }\end{array}$ & MZUEL114 & 5 & autóctone & nativa \\
\hline 142 & Ageneiosus inermis (Linnaeus, 1766) & NUP3161 & 47 & alóctone & Itaipu \\
\hline 143 & Ageneiosus militaris Valenciennes, 1836 & NUP537 & 30 & alóctone & Itaipu \\
\hline 144 & Ageneiosus ucayalensis Castelnau, 1855 & NUP533 & 28,3 & autóctone* & nativa \\
\hline 145 & Auchenipterus osteomystax (Miranda Ribeiro, 1918) & 4532 & 23 & autóctone* & nativa \\
\hline 146 & Glanidium cesarpintoi Ihering, 1928 & 4570 & 10,5 & autóctone & nativa \\
\hline 147 & Tatia neivai (Ihering, 1930) & MZUEL109 & 5,7 & autóctone & nativa \\
\hline 148 & Trachelyopterus coriaceus Valenciennes, 1840 & 6437 & $18 *$ & autóctone* & nativa \\
\hline \multirow[t]{2}{*}{149} & Parauchenipterus galeatus (Linnaeus, 1766) & 1137 & 22 & autóctone* & nativa \\
\hline & $\begin{array}{l}\text { CALLICHTHYIDAE } \\
\text { Callichthyinae }\end{array}$ & & & & \\
\hline 150 & Callichthys callichthys (Linnaeus, 1758) & 8239 & 16,5 & autóctone* & nativa \\
\hline 151 & Hoplosternum littorale (Hancock, 1828) & 1917 & 15,8 & autóctone* & nativa \\
\hline 152 & Lepthoplosternum pectorale (Boulenger, 1895) & NUP4780 & 6 & autóctone* & nativa \\
\hline \multirow[t]{2}{*}{153} & Megalechis personata (Ranzani, 1841) & 8517 & 12,4 & alóctone & Itaipu \\
\hline & Corydoradinae & & & & \\
\hline 154 & Aspidoras fuscoguttatus Nijssen \& Isbrücker, 1976 & 5782 & 3,8 & autóctone & nativa \\
\hline 155 & Aspidoras lakoi Miranda-Ribeiro, 1949 & MNRJ5292 & 4 & autóctone & nativa \\
\hline 156 & Corydoras aeneus (Gill, 1858) & 5786 & 7,5 & autóctone* & nativa \\
\hline 157 & Corydoras difluviatilis Britto \& Castro, 2002 & 6417 & 4,7 & autóctone & nativa \\
\hline 158 & Corydoras ehrhardti Steindachner, 1910 & MCP17803 & 4,1 & autóctone* & nativa \\
\hline 159 & Corydoras flaveolus Ihering, 1911 & 8448 & 3,4 & autóctone & nativa \\
\hline 160 & Corydoras nattereri Steindachner, 1877 & 6935 & 5,4 & autóctone* & nativa \\
\hline 161 & Corydoras paleatus (Jenyns, 1842) & MZUEL612 & 5,9 & autóctone* & nativa \\
\hline \multirow[t]{2}{*}{162} & Scleromystax macropterus (Regan, 1913) & MCP17810 & 8,7 & autóctone* & nativa \\
\hline & Cetopsidae & & & & \\
\hline \multirow[t]{2}{*}{163} & Cetopsis gobioides Kner, 1857 & 8715 & 10,9 & autóctone* & nativa \\
\hline & Clariidae & & & & \\
\hline \multirow[t]{2}{*}{164} & Clarias gariepinus (Burchell, 1822) & NUP2618 & $170 *$ & exótica & piscicultura \\
\hline & Doradidae & & & & \\
\hline 165 & Platydoras armatulus (Valenciennes, 1840) & NUP1052 & 20 & alóctone & Itaipu \\
\hline 166 & Pterodoras granulosus (Valenciennes, 1821) & 4943 & $70 *$ & alóctone & Itaipu \\
\hline 167 & Oxydoras eigenmanni Boulenger, 1895 & NUP4424 & 9,7 & alóctone & Itaipu \\
\hline 168 & Rhinodoras dorbignyi (Kner, 1855) & 7678 & $50 *$ & autóctone* & nativa \\
\hline \multirow[t]{2}{*}{169} & Trachydoras paraguayensis (Eigenmann \& Ward, 1907) & 4872 & 10,4 & alóctone & Itaipu \\
\hline & Heptapteridae & & & & \\
\hline 170 & Cetopsorhamdia iheringi Schubart \& Gomes, 1959 & 5784 & 10,6 & autóctone & nativa \\
\hline 171 & Chasmocranus brachynema Gomes \& Schubart, 1958 & 7974 & 13,1 & autóctone & nativa \\
\hline 172 & Heptapterus multiradiatus Ihering, 1907 & MZUSP4517 & 9,6 & autóctone & nativa \\
\hline 173 & Heptapterus mustelinus (Valenciennes, 1835) & NUP2500 & 20,9 & alóctone & Itaipu \\
\hline 174 & Imparfinis borodini Mees \& Cala, 1989 & 7973 & 15,7 & autóctone & nativa \\
\hline 175 & Imparfinis mirini Haseman, 1911 & 5486 & 8,5 & autóctone & nativa \\
\hline 176 & Imparfinis piperatus Eigenmann \& Norris, 1900 & 7026 & 3,2 & autóctone* & nativa \\
\hline 177 & Imparfinis schubarti (Gomes, 1956) & 3688 & 9,3 & autóctone & nativa \\
\hline 178 & Phenacorhamdia tenebrosa (Schubart, 1964) & 7695 & 6,7 & autóctone & nativa \\
\hline 179 & Phenacorhamdia unifasciata Britski, 1993 & 10447 & 6,2 & autóctone & nativa \\
\hline 180 & Pimelodella avanhandavae Eigenmann, 1917 & 1189 & $9,6^{*}$ & autóctone & nativa \\
\hline 181 & Pimelodella boschmai Van der Stigchel, 1964 & MZUSP2256 & 10 & autóctone & nativa \\
\hline 182 & Pimelodella gracilis (Valenciennes, 1835) & NUP3118 & 17 & autóctone* & nativa \\
\hline 183 & Pimelodella meeki Eigenmann, 1910 & MZUSP28883 & $10,2 *$ & autóctone & nativa \\
\hline 184 & Pimelodella rudolphi Miranda-Ribeiro, 1918 & MZUSP2260Paral & 10,5 & autóctone & nativa \\
\hline 185 & Pimelodella taenioptera Miranda-Ribeiro, 1914 & RMNH23248Hol & 8,8 & alóctone & Itaipu \\
\hline 186 & Rhamdella longipinnis Borodin, 1927 & AMNH8642Hol & 8,8 & autóctone* & nativa \\
\hline 187 & Rhamdia quelen (Quoy \& Gaimard, 1824) & 5743 & 38,7 & autóctone* & nativa \\
\hline 188 & Rhamdiopsis microcephala (Lütken, 1874) & 8600 & 7,8 & autóctone* & nativa \\
\hline 189 & Taunaya bifasciata (Eigenmann \& Norris, 1900) & MZUSP28851 & 14 & autóctone & nativa \\
\hline
\end{tabular}


Tabela 1. Continuação...

\begin{tabular}{|c|c|c|c|c|c|}
\hline & Espécie & Voucher $^{1}$ & Tam. $(\mathbf{c m})$ & Origem $^{2}$ & Ocorrência $^{3}$ \\
\hline \multicolumn{6}{|c|}{ LORICARIIDAE } \\
\hline \multicolumn{6}{|c|}{ Ancistrinae } \\
\hline 190 & Ancistrus cirrhosus (Valenciennes, 1836) & NUP2502 & 8,9 & autóctone* & nativa \\
\hline 191 & Megalancistrus parananus (Peters, 1881) & 4493 & $60 *$ & autóctone* & nativa \\
\hline \multicolumn{6}{|c|}{ Hypoptopomatinae } \\
\hline 192 & Corumbataia cuestae Britski, 1997 & 8031 & 3,3 & autóctone & nativa \\
\hline 193 & Hisonotus depressicauda (Miranda-Ribeiro, 1918) & 8466 & 5 & autóctone & nativa \\
\hline 194 & Hisonotus depressinotus (Miranda-Ribeiro, 1918) & MZUSP2156Hol & 3 & autóctone & nativa \\
\hline 195 & Hisonotus francirochai (Ihering, 1928) & 6451 & 3,6 & autóctone & nativa \\
\hline 196 & Hisonotus insperatus Britski \& Garavello, 2003 & 5377 & 3 & autóctone & nativa \\
\hline 197 & Hisonotus paulinus (Regan, 1908) & 3958 & 4 & autóctone & nativa \\
\hline 198 & Microlepidogaster perforatus Eigenmann \& Eigenmann, 1889 & 8750 & 5 & autóctone & nativa \\
\hline 199 & Otothyropsis marapoama Ribeiro; Carvalho \& Melo, 2005 & 7887 & 3,8 & autóctone & nativa \\
\hline 200 & Pseudotocinclus tietensis (Ihering, 1907) & 6197 & 6 & autóctone & nativa \\
\hline \multicolumn{6}{|c|}{ Hypostominae } \\
\hline 201 & Hypostomus albopunctatus (Regan, 1908) & NUP1761 & 27 & autóctone & nativa \\
\hline 202 & Hypostomus ancistroides (Ihering, 1911) & 1429 & 21 & autóctone & nativa \\
\hline 203 & Hypostomus brevis (Nichols, 1919) & 1451 & 7,4 & autóctone & nativa \\
\hline 204 & Hypostomus cochliodon Kner, 1854 & NUP856 & 23 & alóctone & desconhecida \\
\hline 205 & Hypostomus commersonii Valenciennes, 1836 & MZUSP21817 & 42,5 & alóctone & desconhecida \\
\hline 206 & Hypostomus dlouhyi Weber, 1985 & NUP2560 & 24,5 & alóctone & desconhecida \\
\hline 207 & Hypostomus fluviatilis (Schubart, 1964) & MZUSP44043 & 16,5 & autóctone & nativa \\
\hline 208 & Hypostomus hermanni (Ihering, 1905) & 3102 & 24 & autóctone & nativa \\
\hline 209 & Hypostomus iheringi (Regan, 1908) & 1453 & 11,6 & autóctone & nativa \\
\hline 210 & Hypostomus lexi (Ihering, 1911) & MZUSP2126Hol & 46 & autóctone & nativa \\
\hline 211 & Hypostomus margaritifer (Regan, 1908) & NUP1966 & 33 & autóctone & nativa \\
\hline 212 & Hypostomus meleagris (Marini; Nichols \& La Monte, 1933) & AMNH12246Hol & 30 & autóctone & nativa \\
\hline 213 & Hypostomus microstomus Weber, 1987 & NUP1725 & 24 & autóctone* & nativa \\
\hline 214 & Hypostomus nigromaculatus (Schubart, 1967) & 6425 & 10,2 & autóctone & nativa \\
\hline 215 & Hypostomus paulinus (Ihering, 1905) & BMNH1905.6.9.4Hol & 13,1 & autóctone & nativa \\
\hline 216 & Hypostomus regani (Ihering, 1905) & NUP2286 & $30 *$ & autóctone & nativa \\
\hline 217 & Hypostomus scaphyceps (Nichols, 1919) & AMNH7152Hol & 3,5 & autóctone & nativa \\
\hline 218 & Hypostomus strigaticeps (Regan, 1908) & NUP3140 & 15 & autóctone & nativa \\
\hline 219 & Hypostomus ternetzi (Boulenger, 1895) & NUP1765 & 17,5 & alóctone & desconhecida \\
\hline 220 & Hypostomus tietensis (Ihering, 1905) & MZUSP25176 & 12,5 & autóctone & nativa \\
\hline 221 & Hypostomus topavae (Godoy, 1969) & EEBP315aHol & $70 *$ & autóctone & nativa \\
\hline 222 & Hypostomus variipictus (Ihering, 1911) & 1466 & $37 *$ & autóctone & nativa \\
\hline 223 & Pterygoplichthys anisitsi Eigenmann \& Kennedy, 1903 & 4373 & $42 *$ & autóctone* & nativa \\
\hline 224 & Rhinelepis aspera Spix \& Agassiz, 1829 & 4478 & $33 *$ & autóctone* & nativa \\
\hline \multicolumn{6}{|c|}{ Loricariinae } \\
\hline 225 & Farlowella hahni Meinken, 1937 & 4409 & 20,1 & alóctone & Itaipu \\
\hline 226 & Farlowella oxyrhyncha (Kner, 1853) & NUP1496 & 23 & alóctone & Itaipu \\
\hline 227 & Harttia gracilis Oyakawa, 1993 & MZUSP43267Hol & 10,1 & autóctone & nativa \\
\hline 228 & Loricaria lentiginosa Isbrücker, 1979 & 1561 & 35 & autóctone & nativa \\
\hline 229 & Loricaria piracicabae Ihering, 1907 & MZUSP2182Hol & 17 & autóctone & nativa \\
\hline 230 & Loricaria prolixa Isbrücker \& Nijssen, 1978 & 6312 & 35 & autóctone & nativa \\
\hline 231 & Loricaria simillima Regan, 1904 & 2024 & 18 & alóctone & Itaipu \\
\hline 232 & Loricariichthys platymetopon Isbrücker \& Nijssen, 1979 & 1560 & 30 & alóctone & Itaipu \\
\hline 233 & Loricariichthys rostratus Reis \& Pereira, 2000 & MCP16962Hol & 27,5 & alóctone & Itaipu \\
\hline 234 & Rineloricaria latirostris (Boulenger, 1900) & 3420 & $36^{*}$ & autóctone & nativa \\
\hline 235 & Rineloricaria pentamaculata Langeani \& Araújo, 1994 & 2850 & 12,3 & autóctone & nativa \\
\hline \multicolumn{6}{|c|}{ Neoplecostominae } \\
\hline 236 & $\begin{array}{l}\text { Isbrueckerichthys calvus Jerep; Shibatta; Pereira \& Oyakawa, } \\
2006\end{array}$ & MZUEL3714Hol & 90,2 & autóctone & nativa \\
\hline 237 & $\begin{array}{l}\text { Isbrueckerichthys saxicola Jerep; Shibatta; Pereira \& } \\
\text { Oyakawa, } 2006\end{array}$ & MZUEL3716Hol & 87,7 & autóctone & nativa \\
\hline 238 & Neoplecostomus paranensis Langeani, 1990 & MZUSP38572Hol & 9,3 & autóctone & nativa \\
\hline 239 & Pareorhina carrancas Bockmann \& Ribeiro, 2003 & 8620 & 4,1 & autóctone & nativa \\
\hline \multicolumn{6}{|c|}{ Pimelodidae } \\
\hline 240 & Hemisorubim platyrhynchos (Valenciennes, 1840) & 4399 & 52,5 & autóctone* & nativa \\
\hline
\end{tabular}


Tabela 1. Continuação...

\begin{tabular}{|c|c|c|c|c|c|}
\hline & Espécie & Voucher $^{1}$ & Tam. $(\mathbf{c m})$ & Origem $^{2}$ & Ocorrência $^{3}$ \\
\hline 241 & Hypophthalmus edentatus Spix \& Agassiz, 1829 & 4489 & 57,5 & alóctone & Itaipu \\
\hline 242 & Iheringichthys labrosus (Lütken, 1874) & 7447 & 25 & autóctone* & nativa \\
\hline 243 & Megalonema platanum (Günther, 1880) & 4580 & 34 & alóctone & desconhecida \\
\hline 244 & Pimelodus fur (Lütken, 1874) & 4568 & 25 & alóctone & desconhecida \\
\hline 245 & Pimelodus heraldoi Azpelicueta, 2001 & MZUSP22713Hol & 17,9 & autóctone & nativa \\
\hline 246 & Pimelodus maculatus La Cepède, 1803 & 1273 & 36 & autóctone* & nativa \\
\hline 247 & Pimelodus ornatus Kner, 1858 & 1274 & 38,5 & alóctone & Itaipu \\
\hline 248 & Pimelodus paranaensis Britski \& Langeani, 1988 & MZUSP23089Hol & 26 & autóctone & nativa \\
\hline 249 & Pimelodus platicirris Borodin, 1927 & AMNH8628Hol & _ & autóctone & nativa \\
\hline 250 & Pinirampus pirinampu (Spix \& Agassiz, 1829) & 5716 & $120 *$ & autóctone* & nativa \\
\hline 251 & Pseudoplatystoma corruscans (Spix \& Agassiz, 1829) & 6439 & 114 & autóctone* & nativa \\
\hline 252 & Pseudoplatystoma fasciatum (Linnaeus, 1766) & NUP4485 & $90 *$ & autóctone* & nativa \\
\hline 253 & Sorubim lima (Bloch \& Schneider, 1801) & 4758 & 50,5 & alóctone & desconhecida \\
\hline 254 & Steindachneridion scriptum (Miranda-Ribeiro, 1918) & MZUSP88015 & 77,4 & autóctone* & nativa \\
\hline 255 & Steindachneridion punctatum (Miranda-Ribeiro, 1918) & MZUSP23093 & 63,2 & autóctone* & nativa \\
\hline \multirow[t]{2}{*}{256} & Zungaro jahu (Ihering, 1898) & NUP1194 & 140 & autóctone* & nativa \\
\hline & Pseudopimelodidae & & & & \\
\hline 257 & Microglanis garavelloi Shibatta \& Benine, 2005 & 2993 & 4,2 & autóctone & nativa \\
\hline 258 & Pseudopimelodus mangurus (Valenciennes, 1835) & 5812 & 34,5 & autóctone* & nativa \\
\hline \multirow[t]{2}{*}{259} & Pseudopimelodus aff. pulcher (Boulenger, 1887) & 6173 & $8,7 *$ & autóctone* & nativa \\
\hline & Trichomycteridae & & & & \\
\hline 260 & Parastegophilus paulensis (Miranda Ribeiro, 1918) & 7679 & 5,4 & autóctone* & nativa \\
\hline 261 & Trichomycterus brasiliensis Lütken, 1874 & 8622 & 13,3 & alóctone & desconhecida \\
\hline 262 & Trichomycterus candidus (Miranda-Ribeiro, 1949) & 8591 & 7,5 & autóctone & nativa \\
\hline 263 & Trichomycterus diabolus Bockmann; Casatti \& de Pinna, 2004 & MZUSP78860Hol & 5,4 & autóctone & nativa \\
\hline 264 & Trichomycterus maracaya Bockmann \& Sazima, 2004 & ZUEC6137 & 6,1 & autóctone & nativa \\
\hline 265 & Trichomycterus paolence (Eigenmann, 1917) & MZUSP22752 & 6,8 & autóctone & nativa \\
\hline 266 & Trichomycterus pauciradiatus Alencar \& Costa, 2006 & 8613 & 5,2 & autóctone & nativa \\
\hline \multirow[t]{2}{*}{267} & $\begin{array}{l}\text { Paravandellia oxyptera Miranda Ribeiro, } 1912 \\
\end{array}$ & 6299 & 2,8 & autóctone* & nativa \\
\hline & $\begin{array}{l}\text { ATHERINIFORMES } \\
\text { Atherinopsidae }\end{array}$ & & & & \\
\hline \multirow[t]{3}{*}{268} & Odonthestes bonariensis (Valenciennes, 1835) & NUP2844 & $50 *$ & alóctone & Itaipu \\
\hline & CYPRINODONTIFORMES & & & & \\
\hline & Poeciliidae & & & & \\
\hline 269 & Cnesterodon hypselurus Lucinda \& Garavello, 2001 & MCP22741 & 3,05 & autóctone & nativa \\
\hline 270 & Pamphorichthys hollandi (Henn, 1916) & 8786 & 4,5 & autóctone* & nativa \\
\hline 271 & Phalloceros caudimaculatus (Hensel, 1868) & 2875 & 6,5 & autóctone* & nativa \\
\hline 272 & Phallotorynus fasciolatus Henn, 1916 & MZUSP41373 & 2,9 & autóctone* & nativa \\
\hline 273 & Phallotorynus jucundus Ihering, 1930 & 8380 & 3 & autóctone & nativa \\
\hline 274 & Phallotorynus pankalos Lucinda; Rosa \& Reis, 2005 & MZUSP79671Hol & 2,8 & autóctone & nativa \\
\hline 275 & Phallotorynus victoriae Oliveros, 1983 & MZUSP59257 & 2,3 & autóctone* & nativa \\
\hline 276 & Poecillia reticulata Peters, 1859 & 4775 & $3,5^{*}$ & alóctone & mosquito \\
\hline 277 & Poecillia vivipara Bloch \& Schneider, 1801 & 7614 & $4 *$ & alóctone & mosquito \\
\hline 278 & Xiphophorus helleri Heckel, 1848 & NUP1101 & $14 *$ & alóctone & aquarismo \\
\hline \multirow[t]{2}{*}{279} & Xiphophorus maculatus (Günther, 1866) & 5280 & $6^{*}$ & alóctone & aquarismo \\
\hline & Rivulidae & & & & \\
\hline 280 & Rivulus apiamici Costa, 1989 & MZUSP39980Hol & 3 & autóctone & nativa \\
\hline 281 & Rivulus pictus Costa, 1989 & MNRJ11550Hol & 2,6 & autóctone & nativa \\
\hline 282 & Rivulus pinima Costa, 1989 & MZUSP39978Hol & 3 & autóctone & nativa \\
\hline 283 & Rivulus vittatus Costa, 1989 & MZUSP39980Hol & 3 & autóctone & nativa \\
\hline 284 & Rivulus rossoi Costa, 2005 & UFRJ5976Hol & 2,2 & autóctone & nativa \\
\hline 285 & Rivulus rutilicaudus Costa, 2005 & UFRJ5965Hol & 2,3 & autóctone & nativa \\
\hline 286 & Rivulus scalaris Costa, 2005 & UFRJ5968Hol & 2,9 & autóctone & nativa \\
\hline 287 & Rivulus egens Costa, 2005 & UFRJ5973Hol & 2,6 & autóctone & nativa \\
\hline 288 & Simpsonichthys boitonei de Carvalho, 1959 & MNRJ9012Hol & $5,5^{*}$ & autóctone & nativa \\
\hline 289 & Simpsonichthys parallelus Costa, 2002 & MZUSP57537Hol & $3 *$ & autóctone & nativa \\
\hline \multirow[t]{3}{*}{290} & Simpsonichthys santanae Shibata \& Garavello, 1992 & MZUSP43777Hol & $3 *$ & autóctone & nativa \\
\hline & SYNBRANCHIFORMES & & & & \\
\hline & Synbranchidae & & & & \\
\hline 291 & Synbranchus marmoratus Bloch, 1795 & 7097 & $150 *$ & autóctone* & nativa \\
\hline
\end{tabular}


Tabela 1. Continuação...

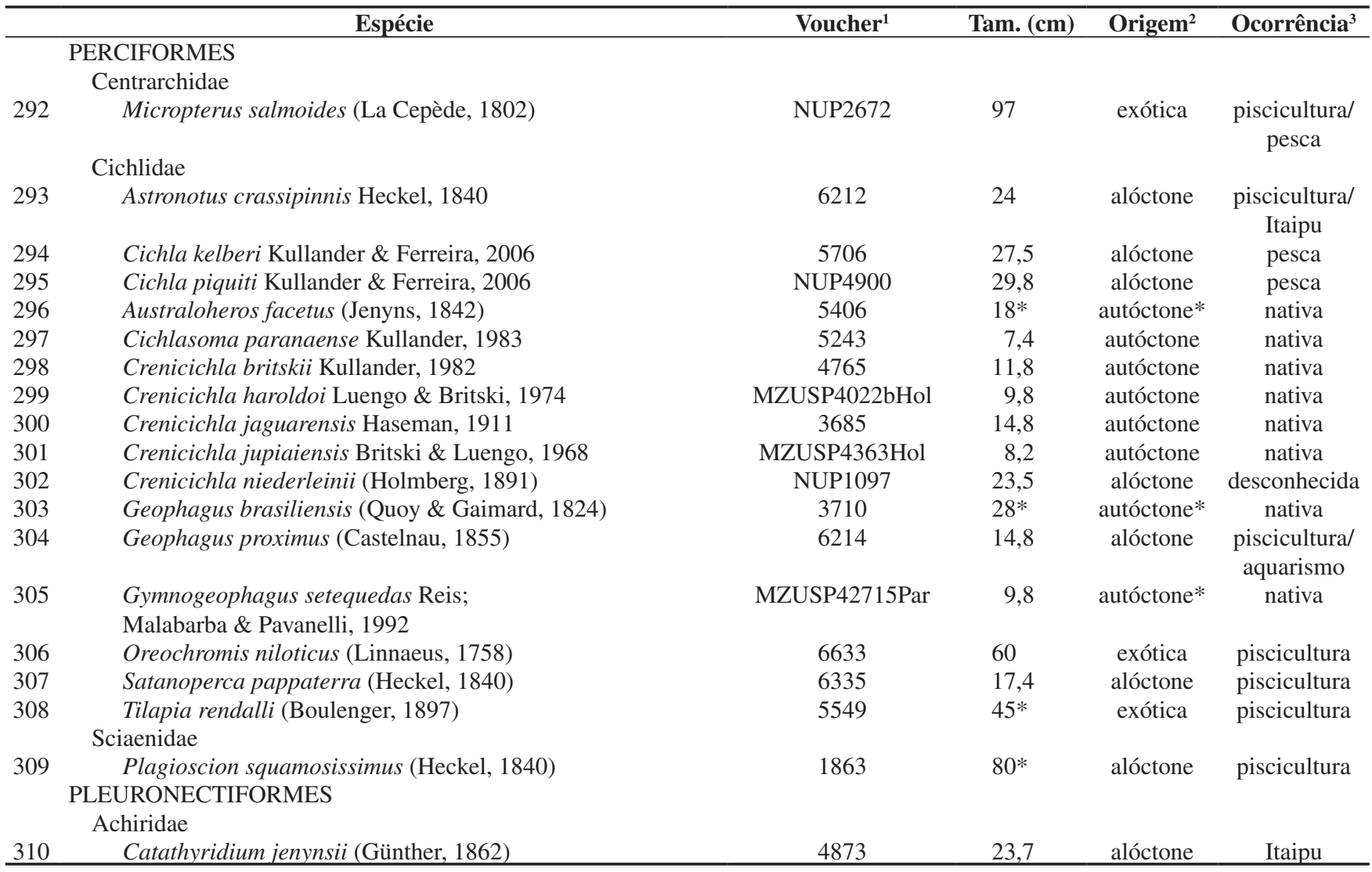

${ }^{1}$ Números sem acrônimo referem-se à coleção de peixes (DZSJRP) da Universidade Estadual Paulista: outras coleções de peixes referidas são American Museum of Natural History (AMNH), Estação Experimental de Biologia de Pirassununga (EEBP), Field Museum of Natural History (FMNH), Laboratório de Ictiologia de Ribeirão Preto, USP (LIRP), Museu de Ciências e Tecnologia da PUCRS (MCP), Museu Nacional da Universidade Federal do Rio de Janeiro (MNRJ), Museu de Zoologia da Universidade Estadual de Londrina (MZUEL), Museu de Zoologia da Universidade de São Paulo (MZUSP), Universidade Estaual de Maringá (NUP), Universidade Federal do Rio de Janeiro (UFRJ), Rijksmuseum van Natuurlijke Historie (RMNH), and Universidade Estadual de Campinas (ZUEC). "Hol", "Par" e "Paral" referem-se, respectivamente, a holotipo, parátipo e paralectótipo; o símbolo \# refere-se à espécie ocorrente no Reservatório de Marimbondo, rio Grande, porém sem exemplar testemunho em coleção.

${ }^{1}$ Numbers without acronym are from the fish collection (DZSJRP) of the Universidade Estadual Paulista; others fish collections are American Museum of Natural History (AMNH), Estação Experimental de Biologia de Pirassununga (EEBP), Field Museum of Natural History (FMNH), Laboratório de Ictiologia de Ribeirão Preto, USP (LIRP), Museu de Ciências e Tecnologia da PUCRS (MCP), Museu Nacional da Universidade Federal do Rio de Janeiro (MNRJ), Museu de Zoologia da Universidade Estadual de Londrina (MZUEL), Museu de Zoologia da Universidade de São Paulo (MZUSP), Universidade Estaual de Maringá (NUP), Universidade Federal do Rio de Janeiro (UFRJ), Rijksmuseum van Natuurlijke Historie (RMNH), and Universidade Estadual de Campinas (ZUEC). "Hol", "Par", and "Paral" refer, respectively, to holotype, paratype, and paralectotype; symbol \# refers to species occurring in the Marimbondo Reservoir, but with voucher specimens not deposited in collections.

${ }^{2}$ Autóctone espécie oriunda do Alto Paraná, asterisco indica localidade-tipo fora do Alto Paraná; alóctone, aquela de outras bacias da região Neotropical; exótica, aquela proveniente de outros continentes.

${ }^{2}$ Autochthonous (autóctone) species from the Upper Paraná, asterisk indicates type locality outside Upper Paraná; allochthonous (alóctone), from other Neotropical basins; exotic (exótica), from other continents.

${ }^{3}$ Nativa, espécie que ocorre naturalmente no sistema; itaipu, espécie que provavelmente dispersou pelo Alto Paraná após a construção do Reservatório de Itaipu; piscicultura, espécie introduzida a partir de pisciculturas, de modo intencional ou acidental; pesca, espécie introduzida para a pesca; aquarismo, espécie de aquário introduzida intencional ou acidentalmente; mosquito, espécie introduzida para o controle de mosquitos; desconhecida, espécie cuja causa de ocorrência é desconhecida.

${ }^{3}$ Native, species occurring naturally in the Upper Paraná system; itaipu, species distributed in the Upper Paraná after the Itaipu dam construction; pisciculture (piscicultura), species intentional or accidentally introduced from aquacultures; fishery (pesca), species introduced for fisheries activities; aquarism (aquarismo), ornamental species intentional or accidentally introduced; mosquito, species introduced to the mosquito control; unknown (desconhecida), species with undetermined cause of occurrence.

cultura, uma itaipu/piscicultura e uma pesca/piscicultura (Tabela 1). As maiores contribuições para o aumento recente do número foram de novas espécies descritas nas últimas duas décadas (49 espécies) (Figura 5) e espécies do baixo Paraná que se dispersaram em função de Itaipu (37 espécies).
O tamanho das espécies variou de 2,2 a 170,5 cm (Figura 6), com uma média de 21,5 cm. A grande maioria das espécies (65\%, 201 de 309 espécies avaliadas) tem porte pequeno, e insere-se na primeira classe de tamanho, até $20,8 \mathrm{~cm} ; 21 \%$ (65 espécies) têm porte médio, variando entre 20,9 e $39,5 \mathrm{~cm}$ de tamanho; todas as 43 espécies res- 


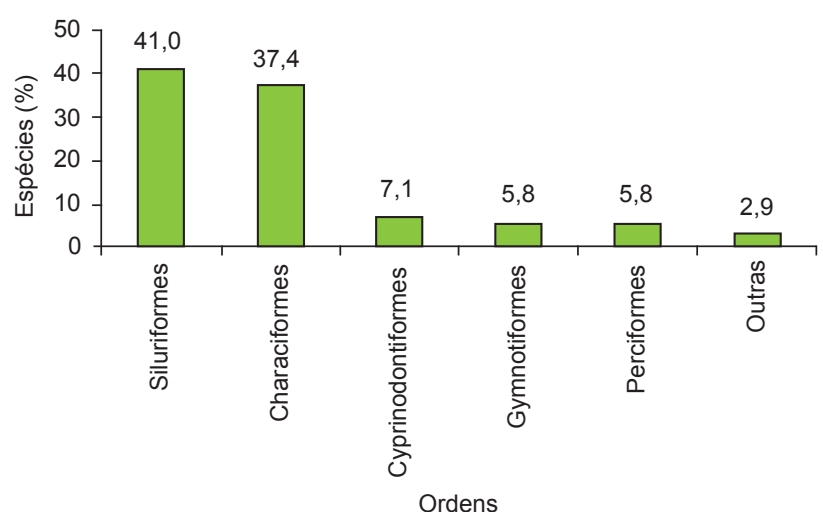

Figura 2. Ictiofauna do Alto Paraná: riqueza de espécies por ordens. Outras - ordens com menos de $1 \%$ da riqueza de espécies: Atheriniformes, Clupeiformes, Cypriniformes, Myliobatiformes, Pleuronectiformes e Synbranchiformes.

Figure 2. Upper Paraná ichthyofauna: species richness by order. Other - orders with less than $1 \%$ of species richness: Atheriniformes, Clupeiformes, Cypriniformes, Myliobatiformes, Pleuronectiformes, and Synbranchiformes.

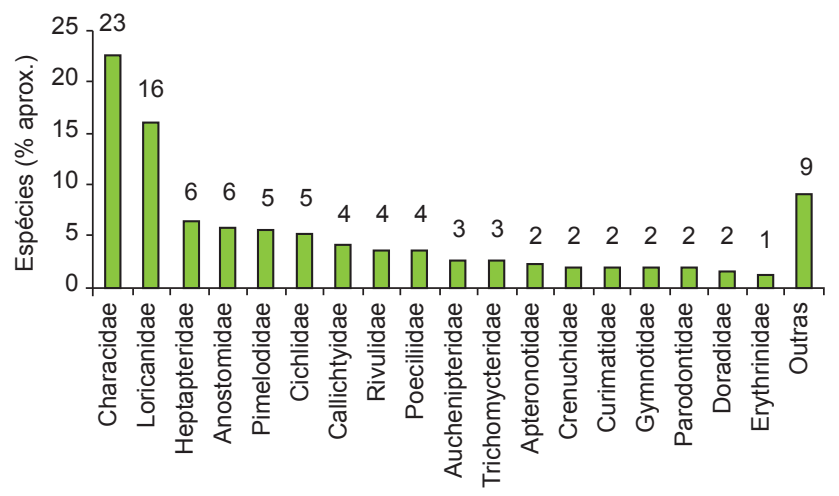

Famílias

Figura 3. Ictiofauna do Alto Paraná: riqueza de espécies por famílias. Outras - famílias com menos de 1\% da riqueza de espécies: Acestrorhynchidae, Achiridae, Aspredinidae, Atherinopsidae, Centrarchidae, Cetopsidae, Clariidae, Clupeidae, Cynodontidae, Cyprinidae, Hemiodontidae, Hypopomidae, Lebiasinidae, Potamotrygonidae, Prochilodontidae, Pseudopimelodidae, Rhamphichthyidae, Sciaenidae, Sternopygidae e Synbranchidae.

Figure 3. Upper Paraná ichthyofauna: species richness by family. Others families with less than $1 \%$ of species richness: Acestrorhynchidae, Achiridae, Aspredinidae, Atherinopsidae, Centrarchidae, Cetopsidae, Clariidae, Clupeidae, Cynodontidae, Cyprinidae, Hemiodontidae, Hypopomidae, Lebiasinidae, Potamotrygonidae, Prochilodontidae, Pseudopimelodidae, Rhamphichthyidae, Sciaenidae, Sternopygidae, and Synbranchidae.

tantes (menos de 15\% do total) foram consideradas de grande porte, variando de 39,6 a $170,0 \mathrm{~cm}$.

O tamanho médio das espécies descritas vem diminuindo ao longo do tempo e três patamares de tamanho são claramente visualizados (Figura 7): do início do período avaliado até a década de 1820, onde foram descritos principalmente peixes de grande porte; da década de 1830 à década de 1890 , principalmente com peixes de porte médio, e no século $\mathrm{XX}$, com a grande maioria das espécies de pequeno porte. Esses dados reforçam que a maioria das primeiras descrições de peixes de água doce da América do Sul restringia-se às espécies de grande porte utilizadas como alimento (Böhlke et al. 1978), e normalmente habitantes da calha dos rios e amplamente distribuídas. Para o Alto Paraná, entretanto, a maior parte dos peixes de porte grande foi

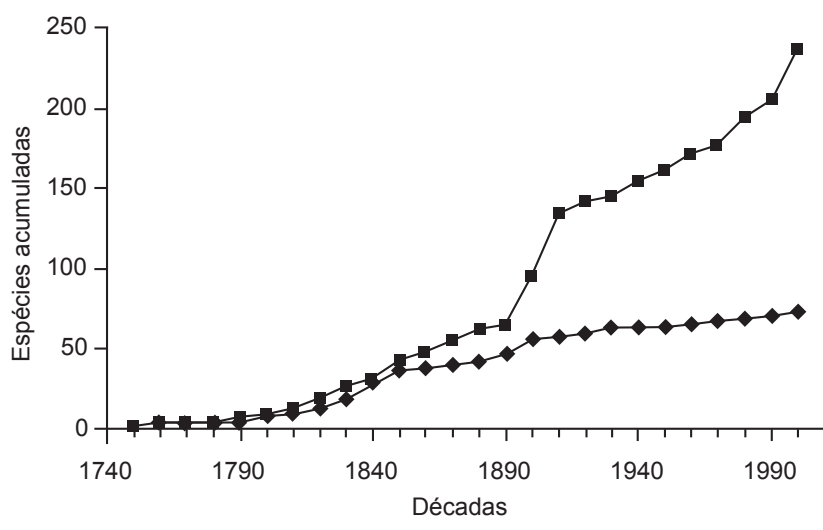

Figura 4. Ictiofauna do Alto Paraná: números acumulados de espécies autóctones (quadrados) e alóctones mais exóticas (losângulos) descritas por década.

Figure 4. Upper Paraná ichthyofauna: accumulated numbers of autochthonous (squares) and allochthonous plus exotic (lozenges) described species by decade.

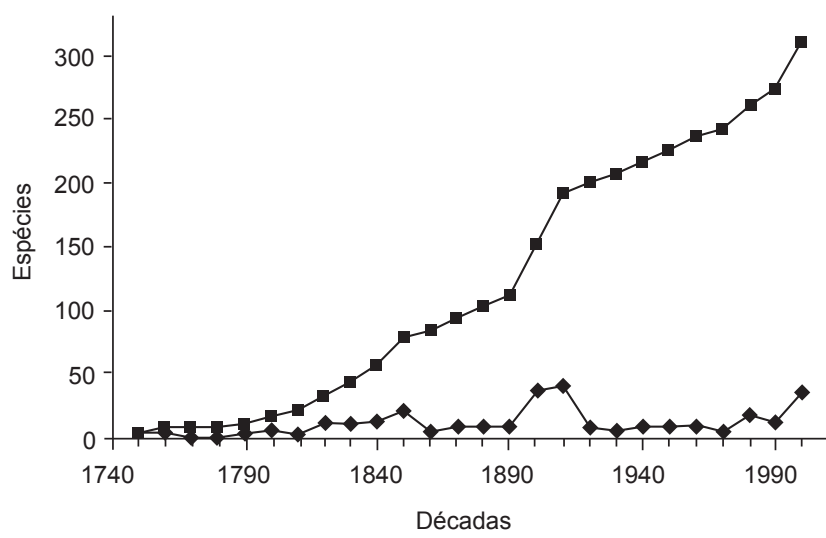

Figura 5. Ictiofauna do Alto Paraná: espécies descritas por década; quadrados (números acumulados), lozangos (números absolutos).

Figure 5. Upper Paraná ichthyofauna: species described by decade; squares (accumulated numbers), lozenges (abslute numbers).

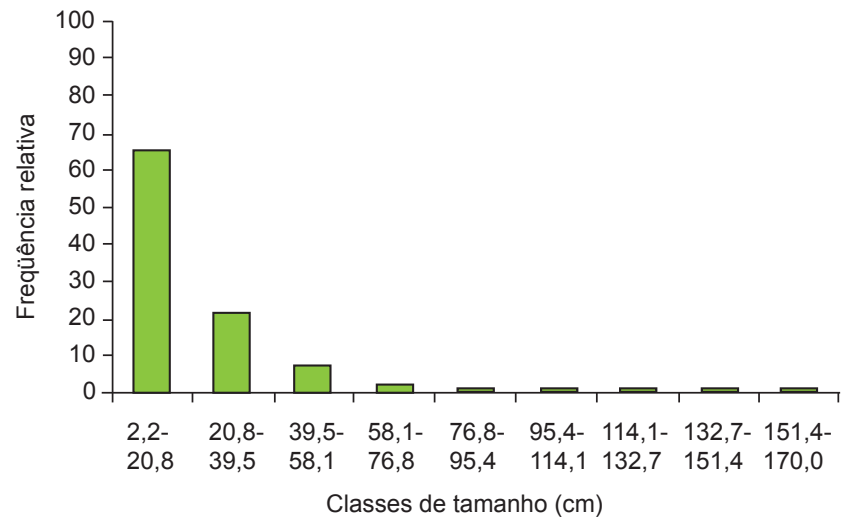

Figura 6. Ictiofauna do Alto Paraná: frequiência relativa do número de espécies por classes de tamanho.

Figure 6. Upper Paraná ichthyofauna: relative frequency of the species number by size classes. 
descrita até a década de 1820 e não até 1866 , como observado por Böhlke et al. (1978) para a América do Sul como um todo.

Desde Lineu (1758), quando foi descrita a primeira espécie, posteriormente referida para o Alto Paraná, a freqüência de descrição de espécies variou de nenhuma a 41 espécies por década (Figura 5), uma média de 11,9 espécies por década ou pouco mais de 1 espécie por ano. Em alguns momentos, entretanto, as descrições aumentaram significativamente, dobrando (década de 1850) ou triplicando (décadas de 1900, 1910 e 2000) (Figura 5). Esses aumentos correspondem principalmente às contribuições de (a) pesquisadores europeus (Louis Agassiz, Marcus Bloch, Georges Cuvier, Albert Günther, Johann Heckel, Rudolf Kner, Christian Lütken, Johannes Reinhardt, Johann Spix, Franz Steindachner e Achille Valenciennes), em meados do século XIX; (b) George Boulenger, Charles Regan e Franz Steindachner (Europa), Carl Eigenmann e Marion Ellis (América do Norte), Rudolf Ihering e Alípio de Miranda Ribeiro (iniciando a pesquisa no Brasil), entre o final do século XIX e o início do século XX, e (c) principalmente de autores brasileiros, muitos dos quais alunos de mestrado e doutorado de Heraldo A. Britski e Naércio A. Menezes (Museu de Zoologia da Universidade de São Paulo) ou alunos de seus alunos, na década atual. O maior aumento (80 novas espécies), notavelmente, deu-se nas duas primeiras décadas do século passado, número ainda não igualado em nenhum outro período. A década atual, entretanto, deverá provavelmente ultrapassar aquelas em número de espécies, pois até o momento já foram descritas 36 espécies (31 autóctones e 5 alóctones) e várias outras estão em processo de descrição (ver abaixo).

Apesar de o Alto Paraná possuir uma das ictiofaunas da América do Sul melhor conhecidas e estudadas, o número espécies aqui registrado está longe de representar a realidade, uma vez que a curva de espécies não mostra nenhuma tendência de estabilização; pelo contrário, apresenta-se numa exponencial ascendente (Figura 5). Por essa razão, diversas descobertas futuras de novos táxons são esperadas no Alto Paraná; uma situação semelhante ocorre também no Baixo Paraná (Luiz R. Malabarba, com. pess.).

Ainda, trabalhos mais recentes evidenciam a singularidade dessa ictiofauna: revisões taxonômicas envolvendo espécies tidas como de ampla distribuição, têm proposto novas espécies para ao menos algumas das bacias envolvidas, e.g. Astyanax altiparanae Garutti \& Britski 2000 em substituição a A. bimaculatus (Lineu 1758) no Alto Paraná, e estão em curso novas descrições para as espécies referidas no Alto Paraná como A. eigenmanniorum (Cope), Bryconamericus iheringii (Boulenger) e Tracheliopterus coriaceus Valenciennes (Adendo e Anexo 1).

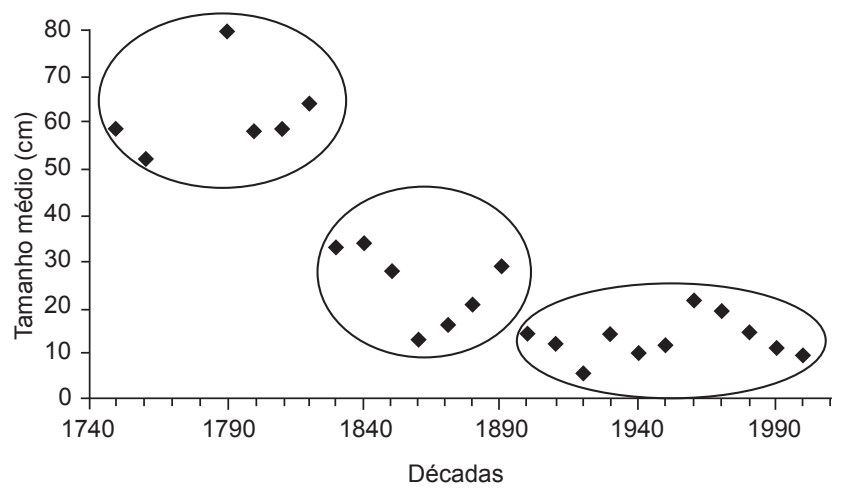

Figura 7. Ictiofauna do Alto Paraná: tamanho médio das espécies (cm) por década.

Figure 7. Upper Paraná ichthyofauna: medium species size $(\mathrm{cm})$ by decade.
Apesar do aumento exponencial de espécies (Figura 5), o número relativo de descrições por autor tem diminuído, com uma variação de 0,9 (década atual) a 11 (década de 1830) (Figura 8), uma média de 2,6 espécies descritas por autor e por década. Entretanto, essa diminuição é atribuída aqui a um maior número de autores estudando essa ictiofauna (Figura 9) e também a um maior número de trabalhos em coautoria, principalmente nas duas décadas mais recentes (Figura 10).

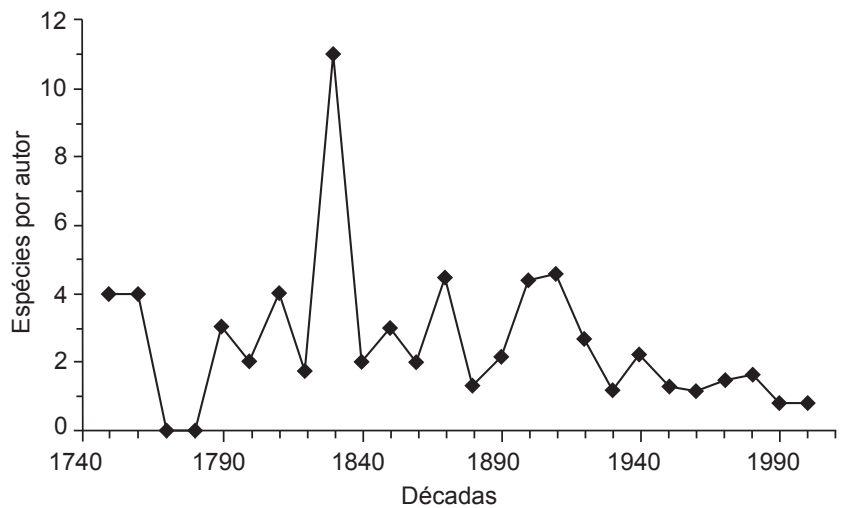

Figura 8. Ictiofauna do Alto Paraná: espécies descritas por autor por década.

Figure 8. Upper Paraná ichthyofauna: described species by author by decade.

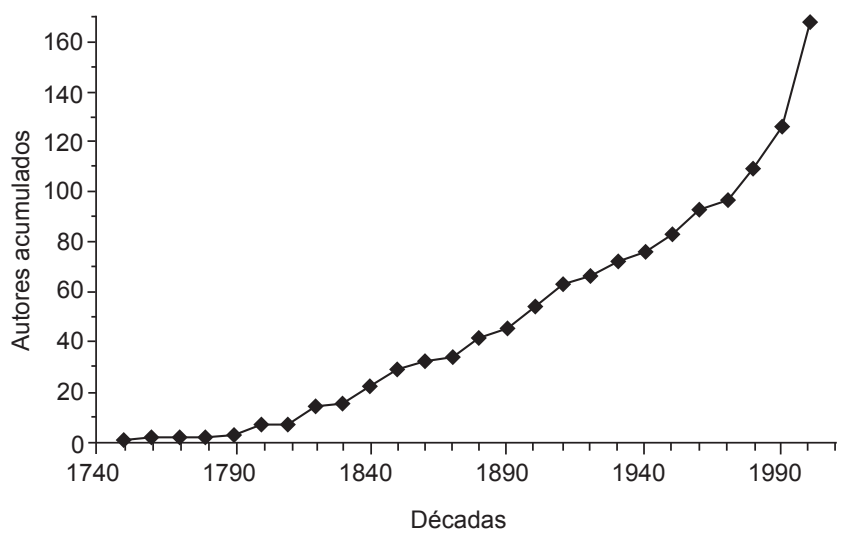

Figura 9. Ictiofauna do Alto Paraná: curva cumulativa de autores por década. Figure 9. Upper Paraná ichthyofauna: accumulative curve of authors by decade.

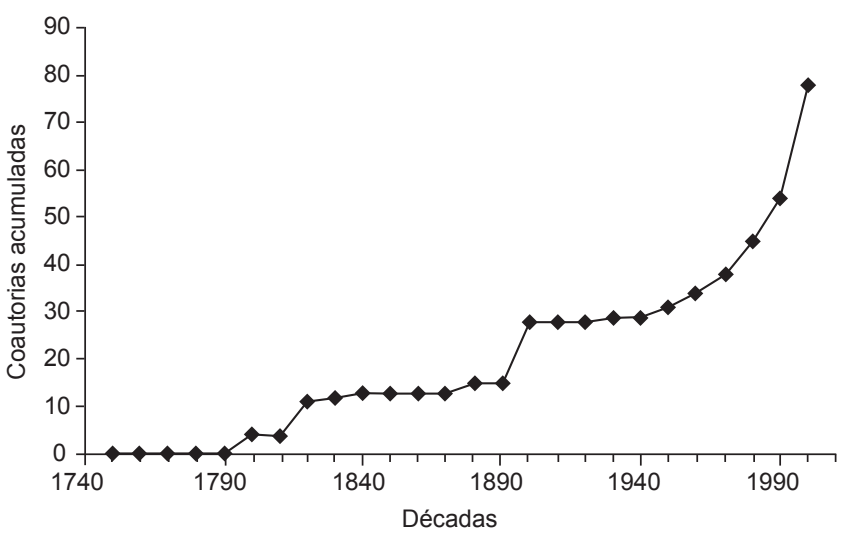

Figura 10. Ictiofauna do Alto Paraná: curva cumulativa de espécies descritas em co-autoria por décadas.

Figure 10. Upper Paraná ichthyofauna: accumulative curve of co-authored species by decades. 
Pelo exposto, não se confirmam na ictiologia sul americana afirmações tais como "much of taxonomy is perceived to be facing a new crisis - a lack of prestige and resources that is crippling the continuing cataloguing of biodiversity" (Godfray 2002) ou "nos últimos anos a taxonomia tem perdido prestígio frente a outras áreas de ciência" (Rapini 2004) e também que a disciplina tem tido menos investimento e o número de praticantes tem decrescido (Valdecasas et al. 2000). Nesse segmento, o número de ictiólogos, taxonomistas e sistematas, na América do Sul tem aumentado (Figura 9) e várias novas espécies, bem como diversos trabalhos de revisão e filogenia para grupos da América do Sul, têm sido propostas. Iniciativas mais amplas estimulando estudos de fauna e flora têm sido igualmente implementadas (e.g., Global Taxonomy Iniciative - GTI, Programa de Taxonomia do MCT, CNPq e CAPES, Programa BIOTA/FAPESP); adicionalmente, o documento gerado em evento paralelo à COP 8 (Salles 2006) reforça o exposto acima ao afirmar que "the international community of taxonomists recognizes an unprecedented set of opportunities to advance biological systematics in unparalleled ways...".

\section{Táxons novos}

Dados compilados, a partir das coleções analisadas e informações de especialistas consultados, permitem estimar a existência de cerca de 50 novas espécies para o Alto Paraná (14,4\% do total geral - espécies referidas mais as novas) (Anexo 1). Esse número encontra-se dentro das estimativas de 6 a $15 \%$ de novas espécies para algumas porções do Alto Paraná, referidos recentemente por Castro et al. (2003, 2004 e 2005). Mantido o ritmo de descrições de novas espécies desta década, aproximadamente cinco por ano, levaríamos mais dez anos para descrever essas 50 espécies já reconhecidas, o que é um tempo demasiadamente longo. Por essa razão é muito importante que a comunidade científica e os órgãos de fomento possam encontrar e viabilizar iniciativas de modo a aumentar esse ritmo, disponibilizando esses novos nomes mais rapidamente, uma vez que grande parte refere-se à espécies de riachos e cabeceiras, hábitats extremamente sujeitos à ação antrópica deletéria.

Dentre essas novas espécies, algumas nunca haviam sido referidas para a drenagem (e.g. espécies de Astyanax, Hasemania, Hemigrammus, Piabina, Moenkhasusia, Characidium, Apareiodon, Microlepidogaster, Neoplecostomus, Harttia, Pamphorichthys e Laetacara) e outras estão sendo propostas para complexos de espécies tidas anteriormente como polimórficas ou de ampla distribuição, em conseqüência de análises morfológicas mais refinadas e séries amostrais maiores e mais completas (e.g. espécies em substituição àquelas anteriormente referidas no Alto Paraná como Astyanax eigenmanniorum, Bryconamericus iheringii, Amaralia hypsiura, Trachelyopterus coriaceus e Phalloceros caudimaculatus). Esse número deve certamente aumentar com estudos adicionais em outras espécies com ampla distribuição (e.g. espécies dos gêneros Brycon, Astyanax, Hemigrammus, Hyphessobrycon, Knodus, Moenkhausia, Characidium, Hoplias, Apteronotus, Gymnotus, Ageneiosus, Pimelodella, Hypostomus, Pimelodus, Pseudopimelodus e Trichomycterus).

Os números apresentados aqui são proporcionais aos pesquisadores atualmente envolvidos com estudos no Alto Paraná (Figura 9) e demonstram, de modo inequívoco, a correlação positiva entre o número de pesquisadores e o número de novas espécies, reforçando a importância de investimentos na formação de ictiólogos, taxonomistas e sistematas. Adicionalmente, a maior parte das novas espécies é proveniente de porções do Alto Paraná dentro do Estado de São Paulo, que comparativamente são aquelas que têm sido alvo de um maior número de estudos. Isso remete à pertinência de se manter esforços de coleta no Estado, mas também de se aumentar os esforços em áreas menos amostradas dos Estados de Goiás, Minas Gerais, Mato Grosso do Sul e Paraná.

Ambientes de riacho são sem dúvida os que apresentam o maior número de novidades, pois 36 das 52 espécies novas potenciais aqui referidas (Anexo 1) são desse tipo de ambiente. Entretanto, coletas em alagadiços e lagoas marginais da região de São José do Rio Preto têm também revelado espécies incomuns em riachos, rios e represas da região, algumas raras e outras aparentemente novas (Araújo \& Langeani 2006, Langeani et al 2007). Adicionalmente, outros ambientes também pouco explorados para espécies de pequeno porte são as áreas marginais de rios e represas que deveriam receber maior atenção.

Importante ressaltar ainda a pertinência de se coletar em riachos de bacias vizinhas ao Alto Paraná, de modo a avaliar a similaridade faunística entre cabeceiras de drenagens distintas e geograficamente próximas. Diversas espécies comuns têm sido referidas para essas porções do Alto Paraná, Ribeira de Iguape, rios costeiros do sudeste brasileiro e São Francisco (Langeani 1989, Oliveira \& Britski 2000, Britto \& Castro 2002, Oyakawa et al. 2005) e coletas mais recentes continuam gerando informações muito relevantes, tais como Serra et al. (2007) sobre a existência de uma típica comunidade de peixes do Alto Tietê (Alto Paraná) em riacho litorâneo do Estado de São Paulo. Uma das espécies mais abundantes naquele riacho, Coptobrycon bilineatus (Ellis), não tem sido mais registrada no Alto Paraná desde a década de 1980, podendo estar extinta nessa drenagem (F.Langeani, obs. pes.). Adicionalmente, Pavanelli \& Britski (1999) referem-se à distinção entre a ictiofauna do Paranaíba e o restante da bacia do Alto Paraná e comentam que isso seria consequiência da ligação entre porções do rio Paranaíba com o sistema do rio Tocantins até o Mioceno.

\section{Adendo}

Adições recentes à ictiofauna do Alto Paraná, não incluídas na presente análise, envolvem: Astyanax bockmanni Vari \& Castro, 2007 (Characiformes, Characidae), novo táxon em substituição à espécie anteriormente identificada no Alto Paraná como Astyanax eigenmanniorum (Cope, 1894) (Vari \& Castro 2007); Pimelodus microstoma Steindachner, 1877 (Siluriformes, Pimelodidae), revalidação de sinônimo-júnior de Pimelodus fur (Lütken, 1874), para aplicação aos exemplares do Alto Paraná (Ribeiro \& Lucena 2007); Corumbataia britskii Ferreira \& Ribeiro, 2007 (Siluriformes, Loricariidae), novo Hypoptopomatinae de riacho afluente do rio Sucuriu, Mato Grosso do Sul; Pituna brevirostrata Costa, 2007 (Cyprinodontiformes, Rivulidae), da planície de inundação do rio Meia Ponte, Goiás; Rivulus illuminatus Costa, 2007 (Cyprinodontiformes, Rivulidae), da planície de inundação do córrego da Queixada, Goiás; Simpsonichthys nigromaculatus Costa, 2007 (Cyprinodontiformes, Rivulidae), da palnície de inundação do rio da Prata, Goiás. Ainda, deve-se adicionar Scoloplax empousa Schaefer, Weitzman \& Britski, 1989 (Siluriformes, Scoloplacidae), família e espécie inadvertidamente não referidas na lista.

\section{Agradecimentos}

A José Luis Birindelli (MZUSP, Anostomidae), Ricardo Campos da Paz (UNIRIO, Gymnotiformes), Carl Ferraris Jr. (Auchenipteridae), Carlos Alberto S. de Lucena (PUCRS, Characinae), Carlos Figueiredo (MNRJ, Poeciliidae, Crenuchidae), Cláudio H. Zawadzki (UEM, Hypostominae), John G. Lundberg (ANSP, Pimelodidae), Luisa Maria Sarmento Soares (MNRJ, Auchenipteridae), Luiz Roberto Malabarba (PUCRS, UFRGS, Cheirodontinae), Marcelo Britto (MNRJ, Callichthyidae), Marilyn Weitzman (USNM, Smithsonian Institution, Lebiasinidae), Mark Sabaj (ANSP, Doradidae), Mônica Toledo-Piza (IBUSP, 
Cynodontidae), Naércio A. Menezes (MZUSP, Acestrorhynchidae, Glandulocaudinae), Paulo H.F. Lucinda (UNITINS, Poeciliidae), Rosana S. Lima (MNRJ, Aphyocharacinae), Sonia Fisch-Muller (MNHN, Genebra, Suíça, Ancistrinae, Loricariidae), Weferson Júnio da Graça (UEM, Cichlidae), Wilson Costa (UFRJ, Rivulidae) pelas valiosas informações sobre grupos dentro de sua especialidade. À equipe do Laboratório de Ictiologia da UNESP, S. J. do R. Preto, Fernando Rogério de Carvalho, Roselene Silva Costa Ferreira, Jane Piton Serra, Flávio Luis Tatsumi, Renato Braz de Araújo, Henrique Figueira Chaves, Diego de Oliveira Tavares, Daiane Simiele, Luiz Gustavo Gorgatto da Silveira, Filipi Césaro Costa e Manoela Maria Ferreira Marinho, pela dedicação aos subprojetos desenvolvidos ou em desenvolvimento. À Fundação de Auxílio à Pesquisa do Estado de São Paulo pelos auxílios concedidos (98/05072-8 para RMCC; 99/05193-2, 04/00545-8 para FL; 01/13340-7 para LC). À FINEP e ao CNPq pelo auxílio ao projeto PRONEX (661058/1997-2, coordenado por Naércio Menezes, para RMCC). Ao CNPq pelas bolsas de Pesquisa de CSP e RMCC. Aos dois revisores anônimos, pelos pareceres bastante críticos e proveitosos.

\section{Referências Bibliográficas}

AB'SABER, N.A. 1998. Megageomorfologia do território brasileiro. In Geomorfologia do Brasil (S.B. Cunha \& A.J.T. Guerra, eds.). Bertand Brasil, Rio de Janeiro, p. 71-106.

AGOSTINHO, A.A. \& JÚLIO JR. H.F. 1999. Peixes da bacia do Alto rio Paraná. In Estudos ecológicos de comunidades de peixes tropicais (R.H. Lowe-McConnell).Edusp, São Paulo, p. 374-400.

AGOSTINHO, A.A. \& GOMES, L.C. 2005. O manejo da pesca em reservatórios da bacia do Alto rio Paraná: avaliação e perspectivas. In Ecologia de reservatórios. impactos potenciais, ações de manejo e sistemas em cascata (M.G. Nogueira, R. Henry \& A. Jorcin, orgs.). Rima Editora, São Carlos, p. 23-55.

AGOSTINHO, A.A., GOMES, L.C. \& PELICICE, F.M. 2007. Ecologia e manejo de recursos pesqueiros em reservatórios do Brasil. Eduem, Maringá, 501p.

ARAÚJO, R.B. \& LANGEANI, F. 2006. Lagoas marginais: viveiros naturais de peixes. O Curumim 15(118):13-16.

BÖHLKE, J.E., WEITZMAN, S.H. \& MENEZES, N.A. 1978. Estado atual da sistemática dos peixes de água doce da América do Sul. Acta Amaz. 8(4):657-677.

BONETTO, A.A. 1986. The Paraná river system. In The ecology of river systems (B.R. Davies \& K.F. Walker, eds.). Dr. W.Junk Publishers, Dordrecht, p. 541-555.

BRITSKI, H.A. 1992. Conhecimento atual das relações filogenéticas de peixes neotropicais. In Situação atual e perspectivas da ictiologia no Brasil (A.A. Agostinho \& E. Benedito-Cecílio, eds.). Documentos do IX Encontro Brasileiro de Ictiologia, Editora da Universidade Estadual de Maringá, Maringá, p. 43-57.

BRITSKI, H.A. \& LANGEANI, F. 1988. Pimelodus paranaensis, sp.n., um novo Pimelodidae (Pisces, Siluriformes) do Alto Paraná, Brasil. Revta Bras. Zool. 5(3):409-417.

BRITSKI, H.A., SATO, Y. \& ROSA, A.B.S. 1988. Manual de identificação de peixes da região de Três Marias (com chaves de identificação para os peixes da bacia do São Francisco). Companhia de Desenvolvimento do Vale do São Francisco - CODEVASF, Brasília.

BRITTO, M.R. \& CASTRO, R.M.C. 2002. New Corydoradine catfish (Siluriformes: Callichthyidae) from the upper Paraná and São Francisco: the sister group of Brochis and most of Corydoras species. Copeia 2002(4):1006-1015.

CASATTI, L., LANGEANI, F. \& CASTRO, R.M.C. 2001. Peixes de riacho do Parque Estadual Morro do Diabo, bacia do Alto Rio Paraná. Biota Neotrop. 1(1,2): http://www.biotaneotropica.org.br/v1n12/pt/ abstract?article+BN00201122001
CASTRO, R.M.C. \& CASATTI, L. 1997. The fish fauna from a small forest stream of the upper Paraná River basin. Ichthyol. Explor. Freshwaters 7(4):337-352.

CASTRO, R.M.C., CASATTI, L., SANTOS, H.F., FERREIRA, K.M., RIBEIRO, A.C., BENINE, R.C., DARDIS, G.Z.P., MELO, A.L.A., ABREU, T.X., BOCKMANN, F.A., CARVALHO, M., GIBRAN, F.Z. \& LIMA, F.C.T. 2003. Estrutura e composição da ictiofauna de riachos do Rio Paranapanema, sudeste e sul do Brasil. Biota Neotrop. 3(1): http:// www.biotaneotropica.org.br/v3n1/pt/abstract?article+BN01703012003

CASTRO, R.M.C., CASATTI, L., SANTOS, H.F., MELO, A.L.A., MARTINS, L.S.F., FERREIRA, K.M., GIBRAN, F.Z., BENINE, R.C., CARVALHO, M., RIBEIRO, A.C., ABREU, T.X., BOCKMANN, F.A., DARDIS, G.Z.P., STOPIGLIA, R. \& LANGEANI, F. 2004. Estrutura e composição da ictiofauna de riachos da bacia do Rio Grande, no Estado de São Paulo, Sudeste do Brasil. Biota Neotrop. 4(1): http://www.biotaneotropica.org. br/v4n1/pt/abstract?article+BN0170402004

CASTRO, R.M.C., CASATTI, L., SANTOS, H.F., VARI, R.P., MELO, A.L.A., MARTINS, L.S.F., ABREU, T.X., BENINE, R.C., GIBRAN, F.Z., RIBEIRO, A.C., BOCKMANN, F.A., CARVALHO, M., PELIÇÃO, G.Z., FERREIRA, K.M., STOPIGLIA, R. \& AKAMA, A. 2005. Structure and composition of the stream ichthyofauna of four tributary rivers of the upper Rio Paraná basin, Brazil. Ichthyol. Explor. Freshwaters 16(3):193-214.

CASTRO, R.M.C. \& MENEZES, N.A. 1998. Estudo diagnóstico da diversidade de peixes do Estado de São Paulo. In Biodiversidade do Estado de São Paulo, Brasil: Síntese do conhecimento ao final do século XX, vertebrados (R.M.C. Castro, ed.). WinnerGraph, São Paulo, p .1-13.

COSTA, W.J.E.M. 2007. Taxononomy of the plesiolebiatine killifish genera Pituna, Plesiolebias and Maratecoara (Teleostei: Cyprinodontiformes: Rivulidae) with descriptions of nine new species.. Zootaxa 1410:1-41.

COX, C.B. \& MOORE, P.D. 2000. Biogeography, an ecological and evolutionary approach. Blackwell Science, London.

ESCHMEYER, W.N. (ed.) 1998. Catalog of fishes. Special publication n ${ }^{\circ} 1$ of the center for Biodiversity Research and Information. California Academy of Sciences, San Francisco.

FERREIRA, K.M. \& RIBEIRO, A.C. 2007. Corumbataia britskii (Siluriformes: Loricariidae: Hypoptopomatinae) a new species from the upper Rio Praná basin, Mato Grosso do Sul, Central Brazil. Zootaxa 1386:59-68.

GÉRY, J. 1969. The fresh-water fishes of South America. In Biogeography and ecology in South America (E.J. Fittkau et al., eds.). Junk, The Hague, p. 828-848.

GODFRAY, H.C.J. 2002. Challenges for taxonomy. Nature 417:17-19.

HUECK, K. \& SEIBERT, P. 1981. Vegetationskarte von Südamerika. Band IIa. Fischer, Sttutgart.

LANGEANI, F. 1989. Ictiofauna do Alto Curso do rio Tietê (SP): taxonomia. Dissertação de Mestrado, Universidade de São Paulo, São Paulo.

LANGEANI, F., CASATTI, L., GAMEIRO, H.S., BELLUCCO-DO-CARMO, A. \& ROSSA-FERES, D.C. 2005a. Riffle and pool fish communities in a large stream of southeastern Brazil. Neotrop. Ichthyol. 3(2):305-311.

LANGEANI, F., LUCENA, Z.M.S., PEDRINI, J. \& TARELHO-PEREIRA, F.J. 2005b. Bryconamericus turiuba, a new species from the upper rio Paraná system (Ostariophysi: Characiformes). Copeia 2005(2):386-392.

LANGEANI, F., SERRA, J.P., CARVALHO, F.R., CHAVES, H.F., FERREIRA, C.P. \& MARTINS, F.O. 2007. Fish, Hasemania crenuchoides Zarske \& Géry, 1999 (Ostariophysi: Characiformes: Characidae): rediscovery and distribution extension in the upper rio Paraná system, Minas Gerais, Brazil. Check List 3(1):119-122.

LATRUBESSE, E.M., STEVAUX, J.C., SANTOS, M.L. \& ASSINE, M.L. 2005. Grandes sistemas fluviais: geologia, geomorfologia e paleohidrologia. In Quaternário no Brasil (C.R.G. Souza, K. Suguio, A.M.S Oliveira \& P.E. Oliveira, eds.). Editora Holos, Ribeirão Preto, p. 276-297.

LINEU, C. 1758. Systema Naturae, Ed. X. (Systema naturae per regna tria naturae, secundum classes, ordines, genera, species, cum characteribus, 
differentiis, synonymis, locis. Tomus I. Editio decima, reformata.) Holmiae. Systema Nat. ed. 10 v. 1: i-ii + 1-824.

LOWE-MCCONNELL, R.H. 1999. Estudos ecológicos de comunidades de peixes tropicais. Edusp, São Paulo.

MALABARBA, M.C. 1998. Phylogeny of fossil Characiformes and paleobiogegraphy of the Tremembé Formation, São Paulo, Brazil. In Phylogeny and classification of Neotropical fishes (L.R. Malabarba, R.E. Reis, R.P. Vari, Z.M.S. Lucena \& C.A.S. Lucena, eds.). EDIPUCRS, Porto Alegre, p. 69-84

MENEZES, N.A., WEITZMAN, S.H. \& BURNS, J.R. 2003. A systematic review of Planaltina (Teleostei: Characiformes: Characidae: Glandulocaudinae: Diapomini) with a description of two new species from the upper rio Paraná, Brazil. Proc. Biol. Soc. Wash. 116(3):557-600.

NELSON, J.S. 2006. Fishes of the world. John Wiley \& Sons, New York.

OLIVEIRA, J.C. \& BRITSKI, H.A. 2000. Redescrição de Taunaya bifasciata (Eigenmann \& Norris, 1900), comb. nova, um bagre enigmático do Estado de São Paulo (Siluriformes, Pimelodidae, Heptapterinae). Pap. Av. Zool. 41:119-133.

OYAKAWA, O.T., AKAMA, A. \& ZANATA, A.M. 2005. Review of the genus Hypostomus Lacépède, 1803 from Ribeira de Iguape basin, with descriptions of a new species (Pisces, Siluriformes, Loricariidae). Zootaxa 921:1-27.

PAVANELLI, C.S. \& BRITSKI, H.A. 1999. Description of a new species of Steindachnerina (Teleostei: Characiformes: Curimatidae) from the upper Rio Paraná basin, Brazil. Ichthyol. Explor. Freshwaters 10:211-216.

RAPINI, A. 2004. Modernizando a taxonomia. Biota Neotrop. 4(1): http://www.biotaneotropica.org.br/v4n1/pt/abstract?point-ofview+BN00204012004

REIS, R.E., KULLANDER, S.O. \& FERRARIS-JR., C.J. (orgs.). 2003. Check list of the freshwater fishes of South and Central America. EDIPUCRS, Porto Alegre.

RIBEIRO, A.C. 2006. Tectonic history and the biogeography of the freshwater fishes from the coastal drainages of eastern Brazil: an example of faunal evolution associated with a divergent continental margin. Neotrop. Ichthyol. 4(3):225-246.
RIBEIRO, A.C., LIMA, F.C.T., RICCOMINI, C. \& MENEZES, N.A. 2006. Fishes of the Atlantic rainforest of Boracéia: tetimonies of the Quaternary fault reactivation within a Neoproterozoic tectonic province in Southeastern Brazil. Ichthyol. Explor. Freshwaters 17(2):157-164.

RIBEIRO, F.R.V. \& LUCENA, C.A.S. 2007. Pimelodus microstoma Steindachner, 1977, a valid species of pimelodid catfish (Siluriformes: Pimelodidae) from the upper rio Paraná drainage. Neotropical Ichthyology 5(1):75-78

SALLES, L.O. (coord). 2006. Outcomes and recommendations of the Meeting on "Biodiversity, the megascience in focus". Relatório apresentado por encontro paralelo à COP 8. Curitiba, Paraná, 15-19 de março de 2006.

SANTOS, G.M. \& FERREIRA, E.J.G. 1999. Peixes da bacia amazônica. In Estudos ecológicos de comunidades de peixes tropicais (R.H. Lowe-McConnell). Edusp, São Paulo, p. 345-373.

SERRA, J.P., CARVALHO, F.R. \& LANGEANI, F. 2007. Ichthyofauna of the rio Itatinga in the Parque das Neblinas, Bertioga, São Paulo: composition and biogeography. Biota Neotrop. 7(1): http://www.biotaneotropica.org. br/v7n1/pt/abstract?article+BN01707012007

SOUZA FILHO, E.E. \& STEVAUX, J.C. 1997. Geologia e geomorfologia do complexo Rio Baía, Curutuba, Ivinheima. In A planície de inundação do Rio Paraná (A.E.A.M. Vazzoler, A.A. Agostinho \& N.S. Hahn, eds.). EDUEM, UEM-NUPELIA, Maringá, p. 3-46.

VALDECASAS, A.G., CASTROVIEJO, S. \& MARCUS, L.F. 2000. Reliance on the citation index undermines the study of biodiversity. Nature 403:698.

VARI, R.P. 1988. The Curimatidae, a lowland neotropical fish family (Pisces: Characiformes); distribution, endemism, and phylogenetic biogeography. In Proceedings of a Workshop on Neotropical Distribution Patterns (W.R Heyer \& P.E Vanzolini, eds). Academia Brasileira de Ciências, Rio de Janeiro, p. 343-377.

VARI, R.P. \& CASTRO, R.M.C. 2007. New species of Astyanax (Ostariophysi: Characiformes: Characidae) from the Upper Rio Paraná System, Brazil. Copeia 2007(1):150-162.

WEITZMAN, S.H. \& MALABARBA, L.R. 1999. Systematics of Spintherobolus (Teleostei: Characidae: Cheirodontinae) from Eastern Brazil. Ichthyol. Explor. Freshwaters 10(1):1-43. 


\section{Anexo 1}

Novas espécies a serem descritas com base em material do Alto rio Paraná, pesquisador responsável e instituição (número de espécies entre parênteses, quando não apenas uma).

\section{Appendix 1}

New species to be described based on specimens from the Upper rio Paraná, responsible researcher and institution (species number in parenthesis when not one).

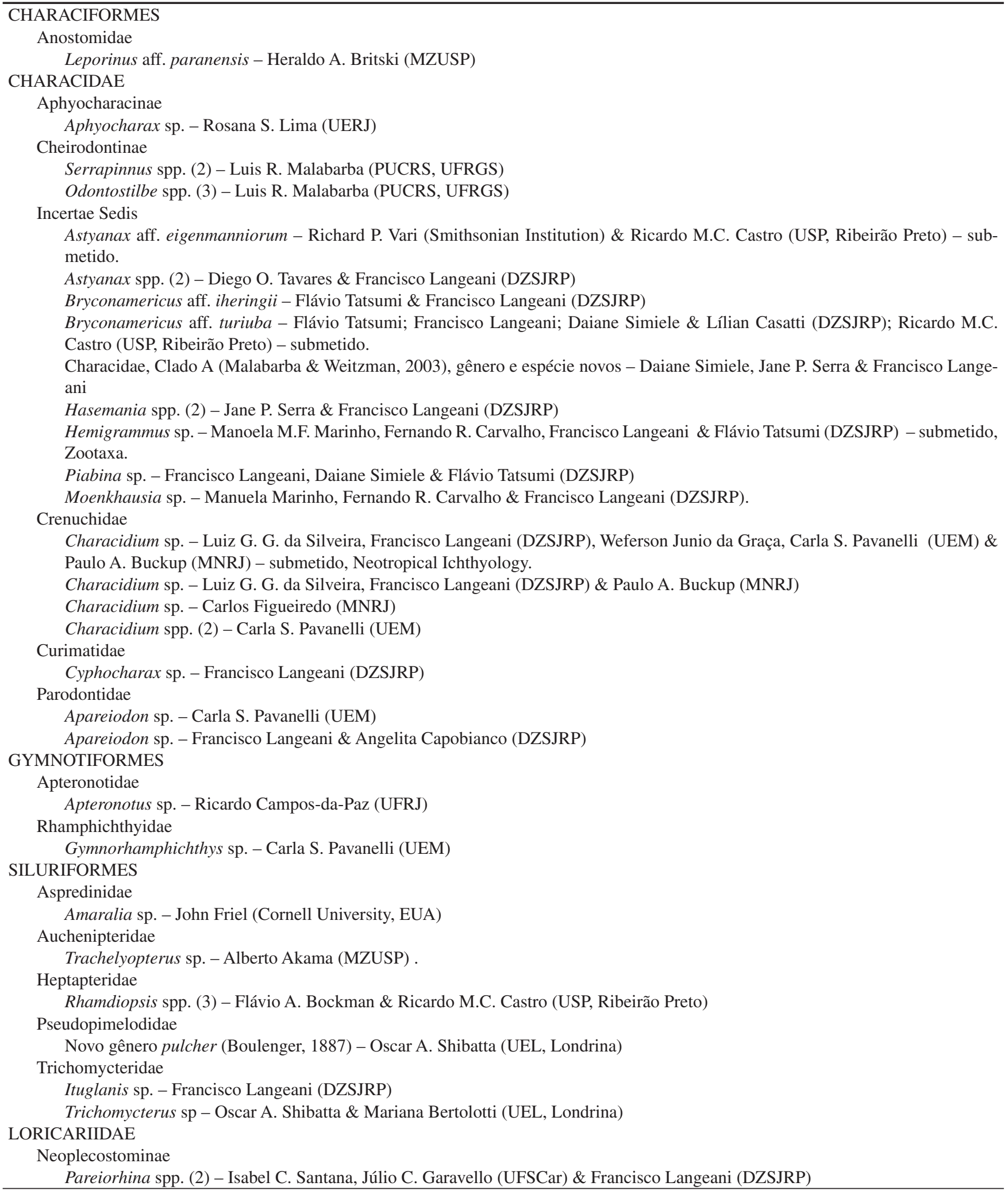


Anexo 1. Continuação...

Neoplecostomus spp. (3) - Cláudio Zawadzki, Carla S. Pavanelli (UEM) \& Francisco Langeani (DZSJRP) - aceito para publicação, Zootaxa

Neoplecostomus sp. - Francisco Langeani (DZSJRP), Flávio A. Bockmann \& Ricardo M.C. Castro (USP, Ribeirão Preto)

Hypoptopomatinae

Microlepidogaster spp. (2) - Filipi Costa \& Francisco Langeani (DZSJRP)

Hypostominae

Hypostomus aff. nigromaculatus - Cláudio Zawadzki (UEM) \& Francisco Langeani (DZSJRP)

Hypostomus sp. - Fernando C. Jerep; Oscar Shibatta (UEL) \& Claudio Zawadzki (UEM)

Loricariinae

Hartia sp. - Osvaldo T. Oyakawa (MZUSP) \& Francisco Langeani (DZSJRP)

CYPRINODONTIFORMES

Poeciliidae

Pamphorichthys sp. - Carlos Figueiredo, Francisco Langeani \& Fernando R. Carvalho (DZSJRP)

Phalloceros spp. (2) - Paulo F. Lucinda (UNITINS)

PERCIFORMES

Cichlidae

Laetacara sp. - Carla S. Pavanelli (UEM), Francisco Langeani \& Fernando R. Carvalho (DZSJRP) 
